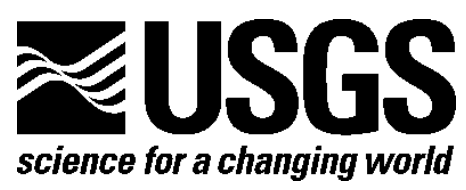

\title{
The Lake Tahoe Basin Land Use Simulation Model
}

By William M. Forney and I. Benson Oldham

Open-File Report 2011-1275

U.S. Department of the Interior

U.S. Geological Survey 


\section{U.S. Department of the Interior \\ KEN SALAZAR, Secretary}

\section{U.S. Geological Survey \\ Marcia K. McNutt, Director}

U.S. Geological Survey, Reston, Virginia: 2011

For product and ordering information:

World Wide Web: http://www.usgs.gov/pubprod

Telephone: 1-888-ASK-USGS

For more information on the USGS-the Federal source for science about the Earth,

its natural and living resources, natural hazards, and the environment:

World Wide Web: http://www.usgs.gov

Telephone: 1-888-ASK-USGS

Suggested citation:

Forney, W.M., and Oldham, I.B., 2011, The Lake Tahoe Basin Land Use Simulation Model: U.S.

Geological Survey Open-File Report 2011-1275, 63 p., available at http://pubs.usgs.gov/of/2011/1275/.

Any use of trade, product, or firm names is for descriptive purposes only and does not imply endorsement by the U.S. Government. Although this report is in the public domain, permission must be secured from the individual copyright owners to reproduce any copyrighted material contained within this report 


\section{Table of Contents}

Abstract

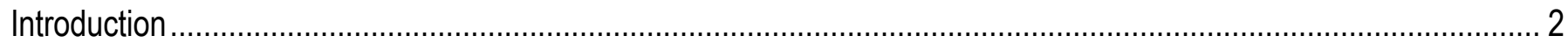

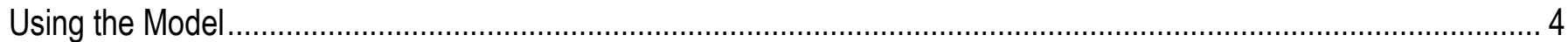

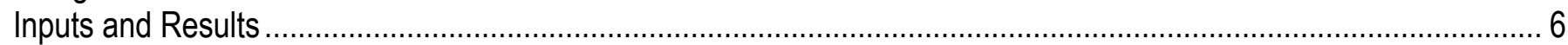

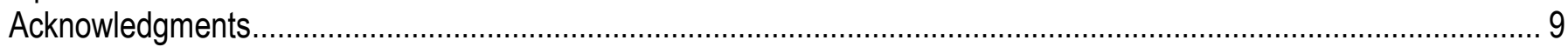

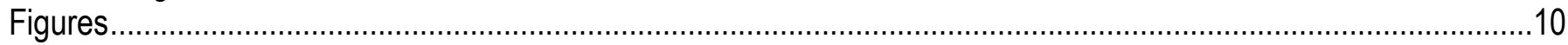

Figure 1. Overview of Lake Tahoe Basin, LUSM inputs, and relevant jurisdictions ...........................................10

Figure 2. Screen shot of the internet GUI for the LUSM showing the list of user variables and values.................. 11

Figure 3. Screen shot of the internet GUI for the LUSM showing the Model parameter values for LUSM

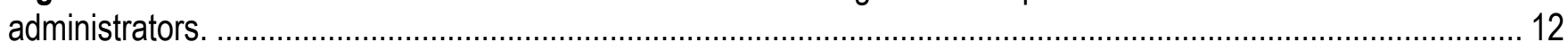

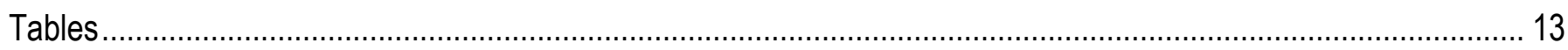

Table 1. User parameters, commodities and their descriptions in the LUSM.............................................. 13

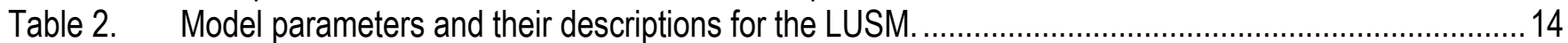

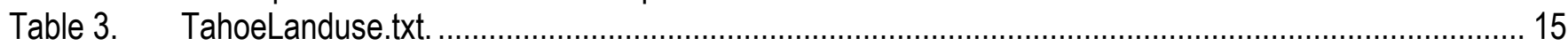

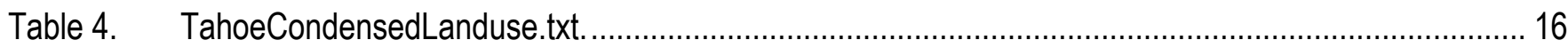

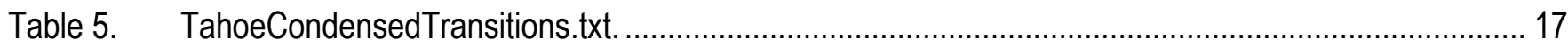

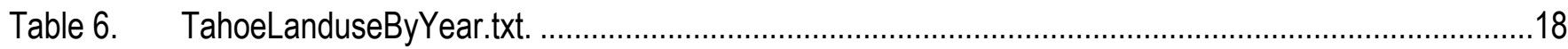

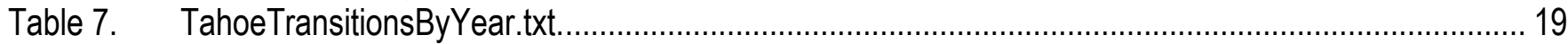

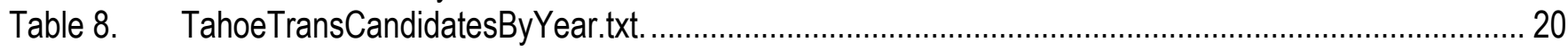

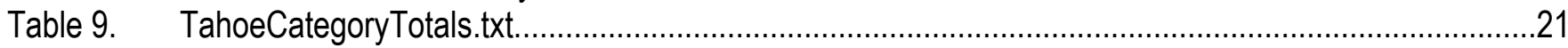

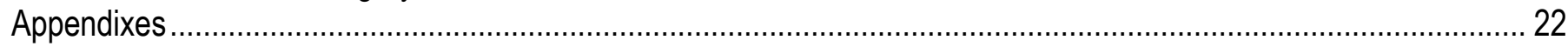

Appendix 1: Tahoe Regional Planning Agency's Parcel Land Use Codes ........................................................22

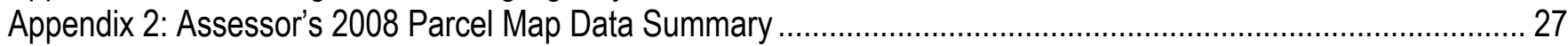

Appendix 3: Samples of Required Databases for the Land Use Simulation Model ................................................. 30

Table 1. Parcel database with Plan Area Statement ID linkage ............................................................. 30

Table 2. Plan Area Statement database ............................................................................................ 31

Appendix 4: Python Code for the Land Use Simulation Model......................................................................... 32 


\section{Acronyms}

\begin{tabular}{|c|c|}
\hline ALLOC_DG & Parcel allocated to Douglas County \\
\hline ALLOC_COMM & Parcel allocated to Commercial square footage \\
\hline ALLOC_EL & Parcel allocated to El Dorado County \\
\hline ALLOC_MFH & Parcel allocated to multiple family housing \\
\hline ALLOC_PL & Parcel allocated to Placer County \\
\hline ALLOC_PL_SENSITIVE & Parcel allocated to the sensitive lots of Placer County \\
\hline ALLOC_SLT $\bar{T}$ & Parcel allocated to the city of South Lake Tahoe \\
\hline ALLOC_TOUR & Parcel allocated to tourist accommodation units \\
\hline ALLOC_- WA & Parcel allocated to Washoe County \\
\hline APN & Assessor's parcel number \\
\hline CTC & California Tahoe Conservancy \\
\hline GUI & Graphical user interface \\
\hline IPES & Individual Parcel Evaluation System \\
\hline OFR & U.S. Geological Survey Open-File Report \\
\hline LUSM & Lake Tahoe Basin Land Use Simulation Model \\
\hline LU & Land Use \\
\hline MFD & Multiple (or multi-) family dwelling (or housing) \\
\hline NDSL & Nevada Division of State Lands \\
\hline PAS & Plan Area Statement \\
\hline RETIRE CTC PRIORITY & Sensitive parcel allocated to California Tahoe Conservancy \\
\hline RETIRE_CTC_SECONDARY & Non-sensitive parcel allocated to California Tahoe Conservancy \\
\hline RETIRE_NEV_PRIORITY & Sensitive parcel allocated to Nevada State Lands \\
\hline RETIRE_NEV_SECONDARY & Non-sensitive parcel allocated to Nevada State Lands \\
\hline RETIRE_TRPĀPRIORITY & Sensitive parcel allocated to Tahoe Regional Planning Agency \\
\hline RETIRE_TRPA_SECONDARY & Non-sensitive parcel allocated to Tahoe Regional Planning Agency \\
\hline RETIRE_USFS_PRIORITY & Sensitive parcel allocated to U.S. Forest Service \\
\hline RETIRE_USFS_SECONDARY & Non-sensitive parcel allocated to U.S. Forest Service \\
\hline SFD & Single family dwelling \\
\hline TAU & Tourist accommodation unit \\
\hline TIIMS & Tahoe Integrated Information Management System \\
\hline TRPA & Tahoe Regional Planning Agency \\
\hline USFS & U.S. Forest Service \\
\hline USGS & U.S. Geological Survey \\
\hline
\end{tabular}




\title{
The Lake Tahoe Basin Land Use Simulation Model
}

\author{
By William M. Forney and I. Benson Oldham
}

\begin{abstract}
This U.S. Geological Survey Open-File Report describes the final modeling product for the Tahoe Decision Support System project for the Lake Tahoe Basin funded by the Southern Nevada Public Land Management Act and the U.S. Geological Survey's Geographic Analysis and Monitoring Program. This research was conducted by the U.S. Geological Survey Western Geographic Science Center. The purpose of this report is to describe the basic elements of the novel Lake Tahoe Basin Land Use Simulation Model, publish samples of the data inputs, basic outputs of the model, and the details of the Python code. The results of this report include a basic description of the Land Use Simulation Model, descriptions and summary statistics of model inputs, two figures showing the graphical user interface from the web-based tool, samples of the two input files, seven tables of basic output results from the web-based tool and descriptions of their parameters, and the fully functional Python code.
\end{abstract}




\section{Introduction}

The Lake Tahoe Basin Land Use Simulation Model (LUSM), which simulates parcel-based, land-use transitions in a stochastic, spatially constrained, agent-based model, was built to inform land management decisions in a complex regulatory environment in the Lake Tahoe Basin (fig. 1). Building on the experience, knowledge and research of the authors and the other U.S. Geological Survey (USGS) Western Geographic Science Center staff, the LUSM was developed in close collaboration with the Tahoe Regional Planning Agency (TRPA). The LUSM provides to TRPA, Basin resource managers and stakeholders a web-based, 20-year-scenario generation tool to simulate the possible allocation of retired (that is, maintained as open space) and developed parcels, and their subsequent transition to another land use fate. Due to the model's capability to project up to 20 years into the future, a random or stochastic element is included to account for the inherent uncertainty of the future. To give the LUSM more site-specific relevance, the model is constrained by 1) neighborhood characteristics and requirements that are defined by Plan Area Statements ${ }^{1}$ (PAS) and 2) TRPA's land capability systems (that is, the Individual Parcel Evaluation System (IPES) and the Bailey Capability System²). Although the definition of an agent can vary, the agent of change in this agent-based model is the parcel's potential land owner. Using standard software packages, coding languages, and readily available datasets, the final LUSM results include reasonable tabular results that can be translated into map-based outputs with minimal additional effort by knowledgeable geographic information system users.

The LUSM is designed as a constrained, stochastic simulation model. This is based on requests of TRPA, data availability, and the consideration of land-use modeling theory when deterministic

1 Includes community plans, both of which are defined by planners at the TRPA. Available at: http://www.trpa.org/default.aspx?tabid=204

2 TRPA Code of Ordinances, Chapter 37 and Chapter 20, respectively. 
outcomes can be misleading, and future scenarios are important to characterize with an adequate balance of realism and uncertainty. The model is constrained by PAS neighborhood allowances, the initial conditions set by the user, and the physical characteristics of a given parcel. The PAS must allow for the parcel's land use type or real estate commodity — otherwise, no commodities of that type will be allocated to the parcels of that PAS. The stochasticity is created by randomizing certain aspects of the selection process, the order of parcels being considered for transition, and modeling specifications and functionality. Also, an essential component - uncertainty — is generated by performing multiple runs or iterations to obtain a set of outcomes. The multiple iterations provide a count of the number of times a specific parcel transitions to a particular land use type or fate, thereby allowing for a probabilistic estimate of the likelihood of that transition. 


\section{Using the Model}

The model and its database are maintained by the Tahoe Integrated Information Management System (TIIMS) and TRPA, and can be fully executed by way of the internet. The model works on a Servoy platform and Servoy's internet-based Graphical User Interface (GUI), and calls the Postgres databases and Python scripting. A description of the project can be found here: http://www.tiims.org/Science-Research/Environmental-Modeling/TDSS.aspx. The LUSM, with permission obtained from TRPA, can be accessed from this webpage.

Instead of running multiple iterations of allocations in a single year to create the stochastic simulation results - then moving onto the next year until 20 years have been reached - the model runs a single iteration of allocations in a given year, removes the parcels that have been allocated from the vacant pool, and moves on to the next year until 20 years have been reached. Upon reaching the twentieth year, this is considered one, full iteration of the model, then - if multiple iterations are specified - it returns to the start time and begins again. This allows for quicker model runs, and arbitrary stochasticity on an annual basis is reduced as allocation targets are maintained from year to year.

All data used for the model were either obtained directly from the TRPA, or were derived from TRPA and other public documents and datasets. The parcel land use codes of TRPA dictate the level of planning detail and functionality of the LUSM. As detailed in appendix 1, only certain TRPA land use codes are important to the LUSM: vacant (private) land (code 1); single family dwelling (SFD, code 1011); multiple family dwelling (MFD, codes 1005, 1006, and 1007); a representative class (3000) for commercial uses including retail, entertainment, services, light industrial, and wholesale/storage; a representative Tourist Accommodation Unit (TAU)—-specifically the Hotel unit (code 2002); and the Resource Management category open space land (code 6401). Please note that multiple family 
dwellings can also be called multi-family dwellings and housing and the acronym MFD represents them both, and that open space land is land that has been taken off the real estate market for development, or retired. Appendix 2 summarizes the 2008 assessor's parcel map dataset that is currently used in the LUSM, and characterizes the land uses in the Lake Tahoe Basin. As of 2008, sixty-eight percent of the land use is residential, less than 1 percent is tourist, over 2 percent is commercial, and over sixteen percent is open space. It is notable that of 60,376 parcels, only 6,326 parcels remain as vacant; these 6,326 parcels that potentially can transition to a different land use are used in the execution of the LUSM. In terms of area, the Tahoe Basin has over 86,000 hectares designated with some type zoning, of which over 2,750 hectares are vacant and included in the execution of the LUSM. 


\section{Inputs and Results}

The LUSM has two primary inputs: the parcel database and Plan Area Statement (PAS) database (fig. 1). Samples of these inputs and the fields are provided in tables 1 and 2 of appendix 3 . Table 1 provides a list and description of the variables that are available to the user, and figure 2 shows what the graphical user interface (GUI) looks like on the internet. Table 2 provides a list of the model's parameters, which are only available to be changed by certain users with administrative privileges, and figure 3 shows what that GUI looks like on the internet.

The user of the model can execute different initial conditions to generate different future land use patterns. Note that for an individual parcel there are multiple fates, which assist in creating the probability of transitioning. Each commodity type has certain variable values and decision rules that govern its behavior. The results of the LUSM are shown in tables 3 through 9, which represent examples of the primary, basic, tabular outputs of the LUSM. The examples were run with the default values of the model, using two iterations. The default values were derived from existing datasets and communications with TRPA. The seven comma-delimited txt files produced by the web-based model were brought into Microsoft Excel for formatting and display purposes. Each table is titled the way it is provided when downloaded in a .zip file from the internet-based model for the sake of continuity and to facilitate the following documentation.

In table 3, "APN" is assessor's parcel number; "Landuse" is the TRPA Land Use Code (appendix 1); "UsePct" is the percent of the time the model designates this particular land use; "Units" are the density-restricted number of units that can be built on a parcel relevant to SFD, MFD, and TAU; and "CommSF" is the amount of commercial square footage allocated in a particular commercial parcel. In table 4, new variables are: “Category”, a particular combination of land use fates for a given parcel; 
"LU_XXXX", a different formulation of the TRPA land use codes and the values are the percent of the time the model designates this particular Land Use; and "MFHUnits," "TourUnits", and "CommSF," static variables that do not change from one run to another and thus they should be disregarded as useful output.

In table 5, new variables are: “ALLOC_DG," the likelihood that a parcel is allocated to Douglas County for SFD; "ALLOC_EL," the likelihood that a parcel is allocated to Eldorado County for SFD; "ALLOC_PL," the likelihood that a parcel is allocated to Placer County for SFD;

“ALLOC_PL_SENSITIVE," the likelihood that a parcel is allocated to the sensitive lots of Placer County for SFD as related to the IPES threshold and the Vacant Lot Equation"; "ALLOC_WA," the likelihood that a parcel is allocated to Washoe County for SFD; "ALLOC_SLT," the likelihood that a parcel is allocated to the city of South Lake Tahoe for SFD; "ALLOC_MFH," the likelihood that a parcel is allocated to multiple family housing; "ALLOC_TOUR," the likelihood that a parcel is allocated to TAU; "ALLOC_COMM," the likelihood that a parcel is allocated to Commercial square footage; "RETIRE_CTC_PRIORITY," the likelihood that a sensitive parcel (as deemed IPES scores and Bailey scores) is allocated to the retirement program of the California Tahoe Conservancy; "RETIRE_CTC_SECONDARY," the likelihood that a non-sensitive parcel is allocated to the retirement program of the California Tahoe Conservancy; "RETIRE_NEV_PRIORITY," the likelihood that a sensitive parcel is allocated to the retirement program of the Nevada State Lands; "RETIRE_NEV_SECONDARY," the likelihood that a non-sensitive parcel is allocated to the retirement program of the Nevada State Lands; "RETIRE_TRPA_PRIORITY," the likelihood that a sensitive parcel is allocated to the retirement program of the TRPA; "RETIRE_TRPA_SECONDARY," the likelihood that a non-sensitive parcel is allocated to the retirement program of the TRPA; "RETIRE_USFS_PRIORITY," the likelihood that a sensitive parcel is allocated to the retirement

3 TRPA Code of Ordinances, Chapter 37.8 
program of the U.S. Forest Service; "RETIRE_USFS_SECONDARY," the likelihood that a nonsensitive parcel is allocated to the retirement program of the U.S. Forest Service. Retirement programs are land acquisition efforts in the Lake Tahoe Basin. Please note that the jurisdictions of the counties and retirement programs are maintained.

Table 6 represents the number of parcels that transition into a particular land use type in a given year, tracked for the duration of the model run. The column headings Y1 through Y20 designate the year of the transition. Table 7 represents the number of parcels that transition into a particular allocation type in a given year, tracked for the years of the model run. Table 8 details the number of parcels that remain available for transitioning to a particular Allocation type in a given year, tracked for the years of the model run. Table 9 represents the frequency of transition fates for the vacant parcels in the Lake Tahoe Basin. New, previously undefined variables in table 9 include: "Count," representing the frequency with which that combination occurs. Note that Category 1 is the most likely combination that results 1,435 times and represents the outcome that a vacant parcel transitions 100 percent of the time to retired/open space (that is, Land Use Code 6401).

The Python source code for the LUSM can be found in appendix 4. 


\section{Acknowledgments}

This research has been supported by the USGS Geographic Analysis and Monitoring Program and funded by the Southern Nevada Public Land Management Act. The research has been conducted in cooperation with the TRPA. The authors would like to thank Peter Ng, Richard Champion, Mara Tongue, and Susan Benjamin for their thorough reviews, and the USGS Western Geographic Science Center staff who laid the groundwork of this effort, especially Richard Bernknopf, David Halsing, Mark Hessenflow, Sean Devlin, Elizabeth Duffy, Peter Ng, and Michael Gould. We appreciate the collaboration with and support of Neil Crescenti, Leif Larson, and Marie Bledsoe of TRPA. 


\section{Figures}

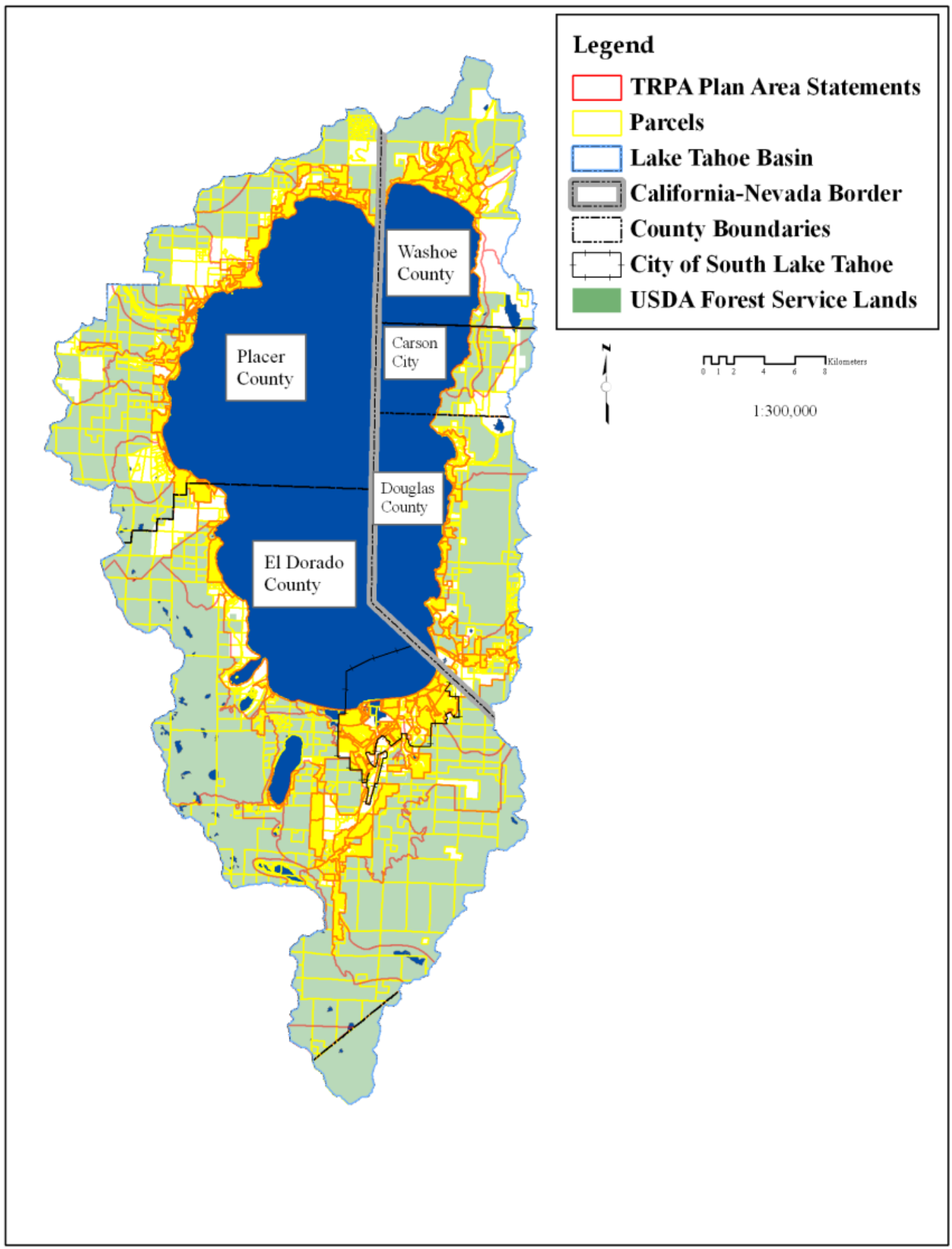

Figure 1. Overview of Lake Tahoe Basin, LUSM inputs, and relevant jurisdictions 


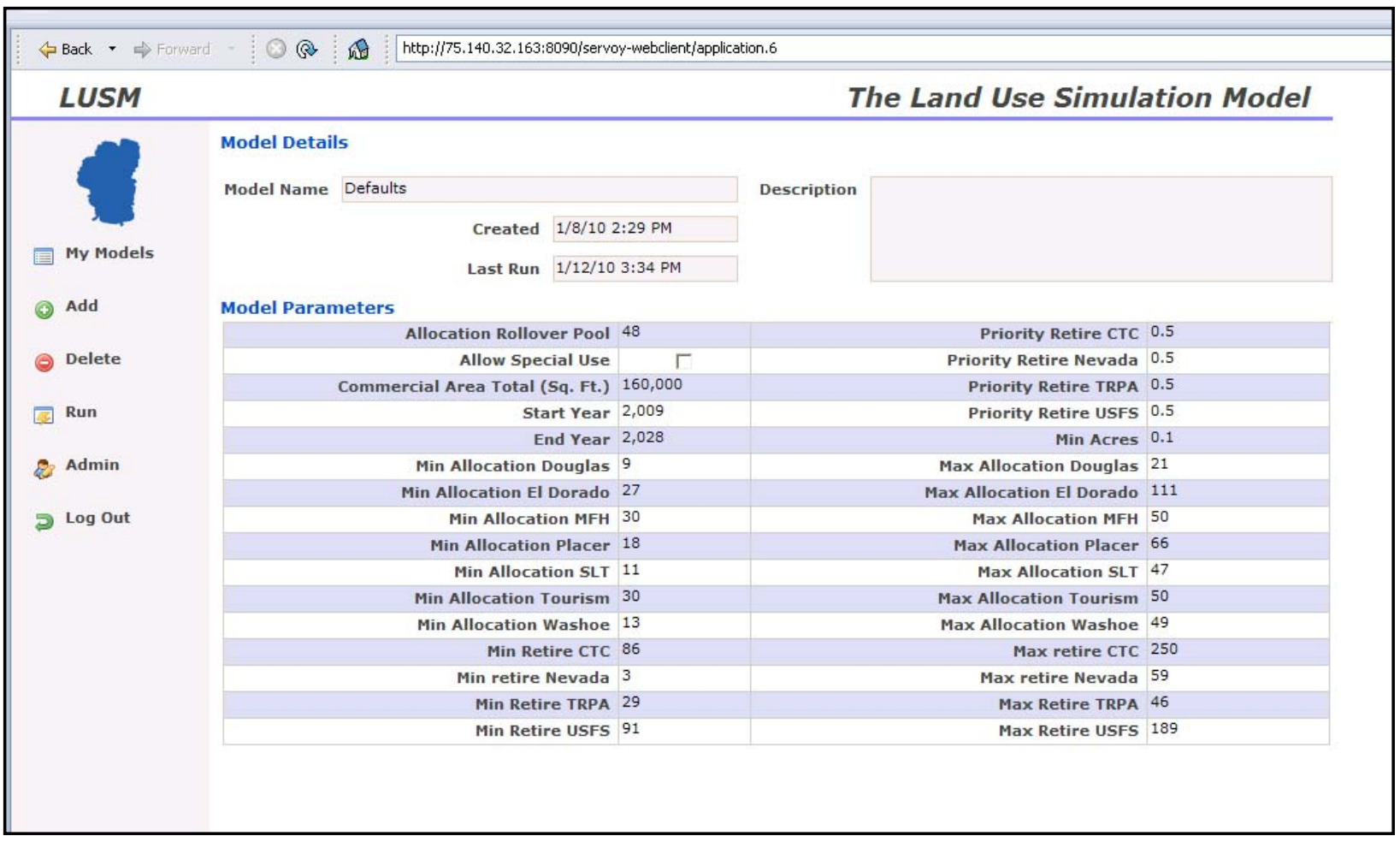

Figure 2. Screen shot of the internet GUI for the LUSM showing the list of user variables and values. 


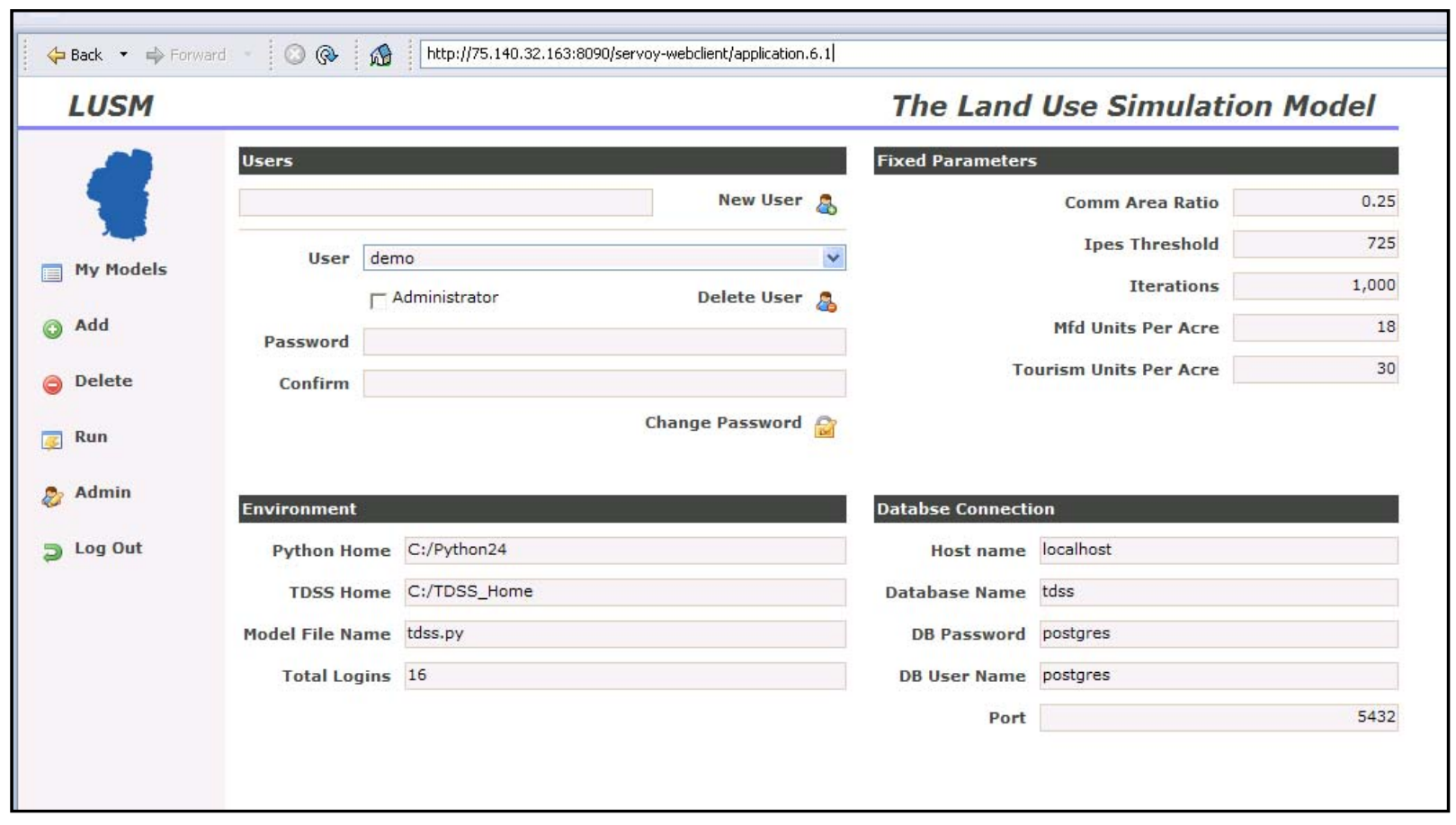

Figure 3. Screen shot of the internet GUI for the LUSM showing the Model parameter values for LUSM administrators.

The Model File Name shows where updates and changes to the Python Code can be made; the Database Name shows where the database can be updated. Also, this is where additional password-protected users and their administrator privileges can be established. 


\section{Tables}

Table 1. User parameters, commodities and their descriptions in the LUSM.

[Single family dwelling (SFD); multi or multiple family dwelling (MFD); tourist accommodation unit (TAU); California Tahoe Conservancy (CTC); Nevada Division of State Lands (NDSL); Tahoe Regional Planning Agency (TRPA); U.S. Forest Service (USFS); plan area statement (PAS)]

\begin{tabular}{|c|c|}
\hline PARAMETER OR COMMODITY NAME & DESCRIPTION OR NOTES \\
\hline \multicolumn{2}{|l|}{ Development } \\
\hline Douglas County-Minimum & Spatial Allocation by County of SFD \\
\hline Douglas County-Maximum & Spatial Allocation by County of SFD \\
\hline Washoe County_-Minimum & Spatial Allocation by County of SFD \\
\hline Washoe County-Maximum & Spatial Allocation by County of SFD \\
\hline El Dorado County-Minimum & Spatial Allocation by County of SFD \\
\hline El Dorado County_-Maximum & Spatial Allocation by County of SFD \\
\hline Placer County-Minimum & Spatial Allocation by County of SFD \\
\hline Placer County-Maximum & Spatial Allocation by County of SFD \\
\hline South Lake Tahoe-Minimum & Spatial Allocation by County of SFD \\
\hline South Lake Tahoe-Maximum & Spatial Allocation by County of SFD \\
\hline Multi-Family Dwelling-Minimum & Spatial Allocation for Basin of MFD \\
\hline Multi-Family Dwelling-Maximum & Spatial Allocation for Basin of MFD \\
\hline Tourist Accommodation Unit-Minimum & Spatial Allocation for Basin of TAU \\
\hline Tourist Accommodation Unit-Maximum & Spatial Allocation for Basin of TAU \\
\hline Commercial Area-Total sq. ft. & Spatial Allocation for Basin of Commercial for the entire model run \\
\hline \multicolumn{2}{|l|}{ Retirement } \\
\hline CTC-Minimum & Spatial Allocation within California \\
\hline CTC-Maximum & Spatial Allocation within California \\
\hline CTC-Priority & Weighting of preferentially sensitive lands to retire \\
\hline NDSL-Minimum & Spatial Allocation within Nevada \\
\hline NDSL-Maximum & Spatial Allocation within Nevada \\
\hline NDSL-Priority & Weighting of preferentially sensitive lands to retire \\
\hline TRPA-Minimum & Spatial Allocation for entire Tahoe Basin \\
\hline TRPA-Maximum & Spatial Allocation for entire Tahoe Basin \\
\hline TRPA-Priority & Weighting of preferentially sensitive lands to retire \\
\hline USFS-Minimum & Spatial Allocation for entire Tahoe Basin \\
\hline USFS-Maximum & Spatial Allocation for entire Tahoe Basin \\
\hline USFS-Priority & Weighting of preferentially sensitive lands to retire \\
\hline \multicolumn{2}{|r|}{ 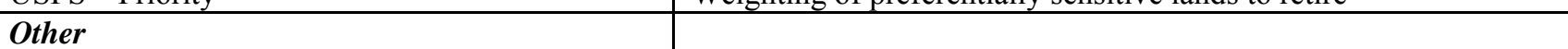 } \\
\hline Allocation Rollover $\mathrm{Pool}^{5}$ & Initial amount in the pool \\
\hline Minimum Size & Minimum size threshold (ac) \\
\hline Special Use Allowance & Inclusion of special uses as designated by individual PASes \\
\hline Starting Year & Beginning year of the model run \\
\hline Ending Year & Final year of the model run \\
\hline
\end{tabular}

TRPA Code of Ordinances, 33.2.A. 
Table 2. Model parameters and their descriptions for the LUSM.

[Multi- or multiple family dwelling (MFD); and Plan Area Statement (PAS)]

\begin{tabular}{|l|l|}
\hline PARAMETER & DESCRIPTION OR NOTES \\
\hline Commercial Area Ratio & $\begin{array}{l}\text { Ratio of parcel size to commercial } \\
\text { footprint area }\end{array}$ \\
\hline IPES threshold & $\begin{array}{l}\text { Value at and below which parcels are } \\
\text { unable to be developed for Placer } \\
\text { County until the Vacant Lot Equation is } \\
\text { satisfied }\end{array}$ \\
\hline Iterations & Number of iterations run by the model \\
\hline MFD Units Per Acre & Density metric for MFD, varies by PAS \\
\hline Tourism Units Per Acre & $\begin{array}{l}\text { Density metric for Tourist } \\
\text { accommodations, varies by PAS }\end{array}$ \\
\hline
\end{tabular}


Table 3. TahoeLanduse.txt.

[This is only a sample of the total parcel-based output. "APN" is assessor's parcel number; "Landuse" is the TRPA Land Use Code; "UsePct" is the percent of the time the model designates this particular land use; "Units" are the density-restricted number of units that can be built on a parcel relevant to single family dwelling, multiple family dwelling, and tourist accommodations; and "CommSF" is the amount of commercial square footage allocated in a particular commercial parcel]

\begin{tabular}{|l|l|r|r|r|}
\hline APN & Landuse & UsePct & Units & CommSF \\
\hline \hline $093-130-026$ & LU_1011 & 0.5 & 1 & 0 \\
\hline $093-130-026$ & LU_6401 & 0.5 & 0 & 0 \\
\hline $093-130-028$ & LU_1011 & 0.5 & 1 & 0 \\
\hline $093-130-028$ & LU_6401 & 0.5 & 0 & 0 \\
\hline $111-120-036$ & LU_1011 & 1 & 1 & 0 \\
\hline $1318-23-810-017$ & LU_1011 & 0.5 & 1 & 0 \\
\hline $1318-23-810-017$ & LU_6401 & 0.5 & 0 & 0 \\
\hline $111-120-034$ & LU_1011 & 0.5 & 1 & 0 \\
\hline $111-120-034$ & LU_6401 & 0.5 & 0 & 0 \\
\hline $1418-03-811-025$ & LU_1011 & 0.5 & 1 & 0 \\
\hline $1418-03-811-025$ & LU_6401 & 0.5 & 0 & 0 \\
\hline $023-501-13$ & LU_6401 & 1 & 0 & 0 \\
\hline $023-103-14$ & LU_6401 & 1 & 0 & 0 \\
\hline $023-103-15$ & LU_1011 & 0.5 & 1 & 0 \\
\hline $023-103-15$ & LU_6401 & 0.5 & 0 & 0 \\
\hline $023-103-16$ & LU_1011 & 0.5 & 1 & 0 \\
\hline $023-103-16$ & LU_6401 & 0.5 & 0 & 0 \\
\hline $023-103-11$ & LU_1011 & 0.5 & 1 & 0 \\
\hline $023-103-11$ & LU_6401 & 0.5 & 0 & 0 \\
\hline $023-512-06$ & LU_6401 & 1 & 0 & 0 \\
\hline $023-512-09$ & LU_6401 & 1 & 0 & 0 \\
\hline $025-271-04$ & LU_1011 & 1 & 1 & 0 \\
\hline $116-110-015$ & LU_1011 & 0.5 & 1 & 0 \\
\hline $116-110-015$ & LU_6401 & 0.5 & 0 & 0 \\
\hline $083-300-015$ & LU_1011 & 1 & 1 & 0 \\
\hline $090-041-009$ & LU_6401 & 1 & 0 & 0 \\
\hline $031-081-10$ & LU_6401 & 1 & 0 & 0 \\
\hline $016-202-20$ & LU_6401 & 1 & 0 & 0 \\
\hline $026-096-05$ & LU_1011 & 0.5 & 1 & 0 \\
\hline
\end{tabular}


Table 4. TahoeCondensedLanduse.txt.

[This is only a sample of the total parcel-based output. "APN" is assessor's parcel number; "Category", a particular combination of fates for a given parcel;

"LU XXXX", a different formulation of the TRPA land use codes and the values are the percent of the time the model designates this particular land use; and

"MFHUnits," "TourUnits", and "CommSF," static variables that do not change from one run to another and thus they should be disregarded as useful output]

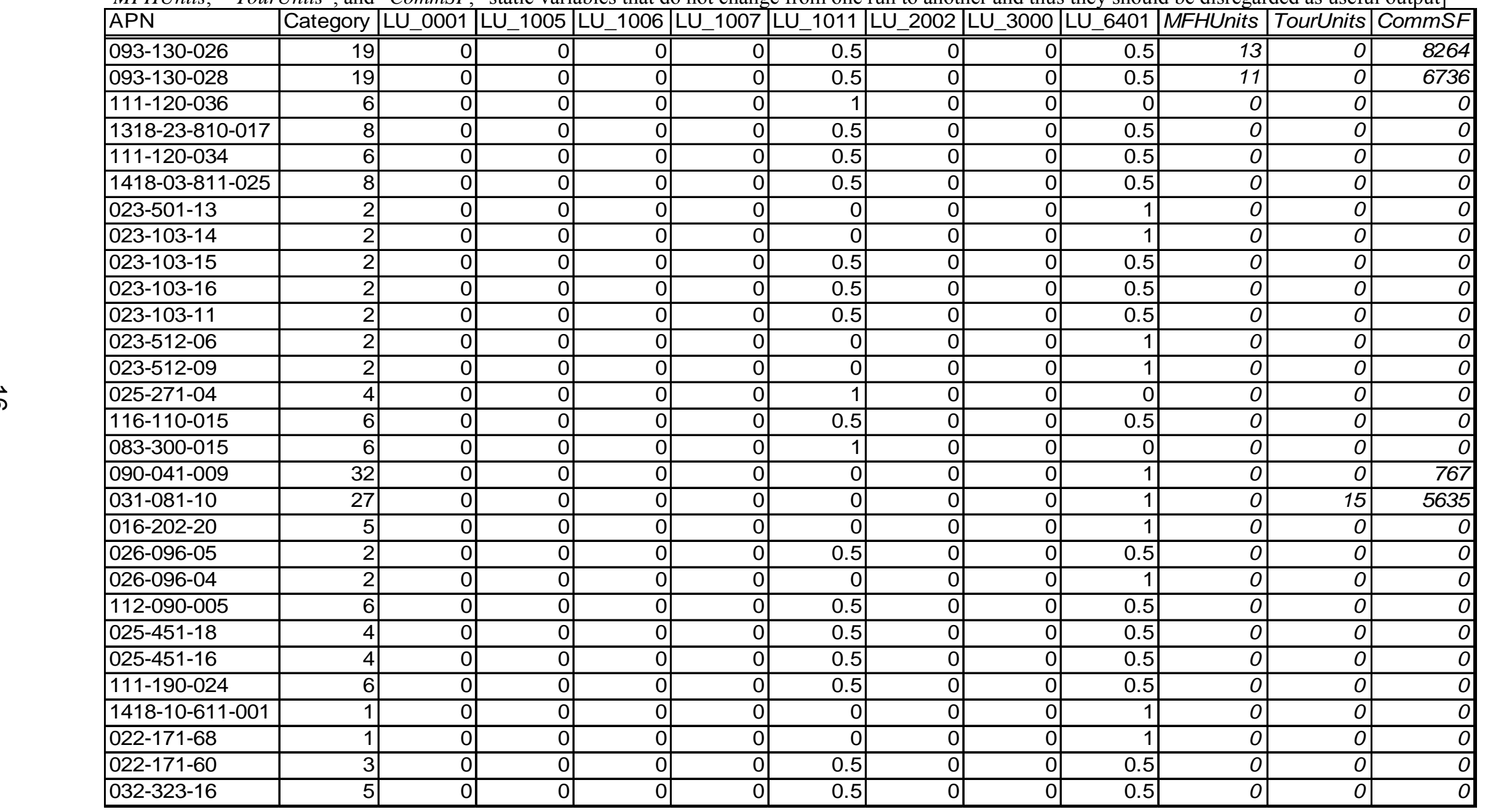


Table 5. TahoeCondensedTransitions.txt.

[This is only a sample of the total parcel-based output. "APN" is assessor's parcel number; "ALLOC_DG," a parcel is allocated to Douglas County;

"ALLOC_EL," a parcel is allocated to Eldorado County; "ALLOC_PL," a parcel is allocated to Placer County; "ALLOC_PL_SENSITIVE," a parcel is allocated to the sensitive lots of Placer County; "ALLOC_WA," a parcel is allocated to Washoe County; "ALLOC_SLT," a parcel is allocated to the city of South Lake Tahoe; "ALLOC_MFH," a parcel is allocated to multiple family housing; "ALLOC_TOUR," a parcel is allocated to Tourist accommodations;

"ALLOC_COMM," a parcel is allocated to commercial square footage; "RETIRE_CTC_PRIORITY," a sensitive parcel is allocated to the retirement program of the California Tahoe Conservancy; "RETIRE_CTC_SECONDARY," a non-sensitive parcel is allocated to the retirement program of the California Tahoe Conservancy; "RETIRE NEV PRIORITY," a sensitive parcel is allocated to the retirement program of the Nevada State Lands;

"RETIRE_NEV_SECONDARY," a non-sensitive parcel is allocated to the retirement program of the Nevada State Lands; "RETIRE_TRPA_PRIORITY," a sensitive parcel is allocated to the retirement program of the TRPA; "RETIRE_TRPA_SECONDARY," a non-sensitive parcel is allocated to the retirement program of the TRPA; "RETIRE_USFS_PRIORITY," a sensitive parcel is allocated to the retirement program of the U.S. Forest Service; "RETIRE_USFS SECONDARY," a non-sensitive parcel is allocated to the retirement program of the U.S. Forest Service]

\begin{tabular}{|c|c|c|c|c|c|c|c|c|c|c|c|c|c|c|c|c|c|}
\hline APN & \begin{tabular}{|l|} 
ALLO \\
C_DG
\end{tabular} & \begin{tabular}{|l|} 
ALLOC \\
EL
\end{tabular} & \begin{tabular}{|l|} 
ALLO \\
C_PL \\
\end{tabular} & $\begin{array}{l}\text { ALLOC_PL-PL- } \\
\text { SENSITIVE }\end{array}$ & $\begin{array}{l}\text { ALLOC } \\
\text { WA }\end{array}$ & $\begin{array}{l}\text { ALLOC } \\
\text { ASLT }\end{array}$ & $\begin{array}{l}\text { ALLOC } \\
\text { MFH }\end{array}$ & \begin{tabular}{|l|} 
ALLOC \\
TOUR
\end{tabular} & $\begin{array}{l}\text { ALLOC_ } \\
\text { COMM }\end{array}$ & \begin{tabular}{|l|} 
RETIRE_CTC \\
PRIORITY
\end{tabular} & $\begin{array}{l}\text { RETTRE_CTC } \\
\text { SECONDARY }\end{array}$ & $\begin{array}{l}\text { RETIRE_NEV } \\
\text { PRIOEITY }\end{array}$ & $\begin{array}{l}\text { RETTRENNEV } \\
\text { SECONDARY }\end{array}$ & \begin{tabular}{|l} 
RETIRE_TRPA \\
PRIORITY
\end{tabular} & $\begin{array}{l}\text { RETIRE_TRPA } \\
\text { SECONDARY }\end{array}$ & $\begin{array}{l}\text { RETIRE_USFS } \\
\text { PRIORITY }\end{array}$ & $\begin{array}{l}\text { RETIRE_USFS_ } \\
\text { SECONDARY }\end{array}$ \\
\hline$\overline{093-130-026}$ & & & 0.5 & & & & & & & & & & & & & & \\
\hline 30-028 & & & 0.5 & & 0 & & 0 & & & & & & & & & & \\
\hline$\frac{111-120-0}{112}$ & 0 & & 1 & $\underline{0}$ & $\underline{0}$ & 0 & 0 & 0 & $\underline{0}$ & & 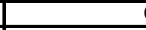 & & & & 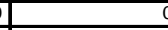 & 0 & \\
\hline$\frac{1318-23-8}{111-28}$ & $\frac{5}{n}$ & & of & $\frac{0}{n}$ & $\frac{0}{n}$ & $\sigma_{0}^{0}$ & 0 & $e_{0}$ & $e_{0}$ & $e_{0}^{0}$ & $\sqrt{n}$ & $\frac{0}{n}$ & & & & 0 & \\
\hline 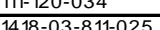 & 0 & & & -0 & -0 & 50 & $\frac{0}{0}$ & 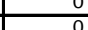 & & 0 & 0. & $\frac{0}{2}$ & 0 & & 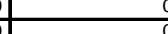 & $\frac{0}{0}$ & \\
\hline$\frac{140}{023-501-13}$ & & & 20 & 0 & 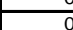 & 0 & $\frac{0}{0}$ & $\frac{0}{0}$ & ce & 0 & -7 & & & & $\frac{0.5}{0.5}$ & $\frac{0}{0}$ & \\
\hline$\frac{023-10:}{020}$ & & & & & & & 0 & & $c^{2}$ & 0 & & & & & & 0 & \\
\hline $023-103-15$ & 0 & & & 0 & 0 & 0.5 & $\overline{0}_{0}$ & & $c_{9}$ & 0 & 7 & & & & $f$ & $\overline{0}$ & \\
\hline & 0 & & & 0 & 0 & 0.5 & 0 & 0 & $c$ & & 0. & & & & 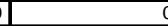 & 0 & \\
\hline 023-103-11 & 0 & 0 & 0 & 0 & a & 0.5 & 0 & 0 & 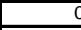 & & $Z$ & & & & 1 & 0 & if \\
\hline$\frac{023-5212}{\frac{02527}{25}}$ & 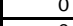 & & 0 & -0 & $-\frac{0}{n}$ & & 0 & & $-a^{2}$ & & 0 & & & & $t$ & $\frac{0}{2}$ & \\
\hline$\frac{0 \angle 3-5 \angle-09}{025-271-04}$ & $\frac{0}{0}$ & & of & 50 & 50 & 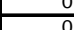 & 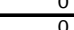 & 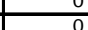 & -6 & -5 & 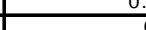 & & - & & 0.8 & $x^{0}$ & \\
\hline $116-110-015$ & 0 & & \begin{tabular}{|l|}
0.5 \\
\end{tabular} & & 0 & 0 & 0 & 0 & 0 & & 0. & & & & & -1 & \\
\hline & 0 & & & 0 & & & & & & & & & & & & & \\
\hline व90-041-009 & 0 & 0 & \begin{tabular}{|l|} 
\\
\end{tabular} & 0 & 0 & 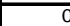 & 0 & 0 & $c_{2}$ & 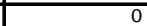 & 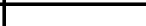 & & & & 5 & 0 & \\
\hline $031-081-10$ & 0 & & O & & 0 & 0 & 0 & & & & & & & & f & 0 & \\
\hline $\begin{array}{l}016-202-20 \\
0\end{array}$ & 0 & 0 & 0 & 0 & 0 & 0 & 0 & 0 & $c^{-1}$ & 0.5 & 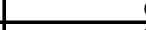 & & & & 4 & 0.5 & \\
\hline$\frac{026-906-05}{20-05}$ & $\sigma_{0}^{0}$ & & & $-\frac{0}{n}$ & $a^{0}$ & 0.5 & 0 & & & & & & & & $-c_{0}$ & & $f$ \\
\hline$\frac{020-900-04}{112-090}$ & $\frac{0}{0}$ & 0 & 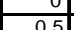 & $\frac{0}{0}$ & $\frac{0}{0}$ & $-a_{0}$ & $\frac{0}{0}$ & $\frac{0}{0}$ & -0 & 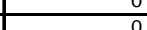 & - & & & & 0 & $\frac{0}{0}$ & \\
\hline $025-451-18$ & & 0.5 & \begin{tabular}{|l|}
0 \\
\end{tabular} & & 0 & 0 & 0 & & 7 & 0 & & & & & 0. & 0 & of \\
\hline & 0 & 0.5 & 0 & 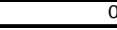 & 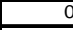 & 0 & 0 & 0 & $Z$ & 0 & - & & & & c & 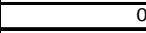 & $f$ \\
\hline$\frac{111-190-0}{11418-0}$ & & & \begin{tabular}{|l|} 
\\
\end{tabular} & $\frac{0}{0}$ & 0 & 0 & 0 & & - & $\frac{0}{0}$ & - & & & & 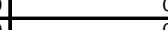 & $\frac{0}{n}$ & 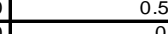 \\
\hline $022-171-68$ & 0 & & 0 & & 0 & 0 & 0 & & & & & & & & t & 0 & \\
\hline$\frac{022-171-60}{032-323-16}$ & & 0 & 0 & 0 & 0 & 0.5 & & & 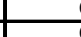 & & & & & & & 0.5 & \\
\hline & & & & & & & & & & & & & & & & & \\
\hline
\end{tabular}


Table 6. TahoeLanduseByYear.txt.

[Y1 through Y20 designate the year of the transition; "LU_XXXX", a different formulation of the TRPA land use codes and the values are the percent of the time the model designates this particular Land Use; and "MFHUnits," "TourUnits", and "CommSF," static variables that do not change from one run to another and thus they should be disregarded as useful output]

\begin{tabular}{|c|c|c|c|c|c|c|c|c|c|c|c|c|c|c|c|c|c|c|c|c|}
\hline Desc & Y1 & Y2 & Y3 & Y4 & Y5 & Y6 & Y7 & Y8 & Y9 & Y10 & Y11 & Y12 & Y13 & Y14 & Y15 & Y16 & Y17 & Y18 & Y19 & Y20 \\
\hline "LU_0001 & - & - & - & - & - & - & - & - & - & - & 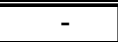 & - & - & - & 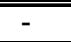 & - & - & - & - & - \\
\hline LU_1005 & 13 & 9 & 12 & 9 & 16 & 9 & 8 & 6 & 8 & 12 & 10 & 4 & - & - & - & - & - & - & - & - \\
\hline LU_1006 & 1 & 2 & 2 & 1 & 2 & 2 & 2 & 2 & 2 & 1 & 3 & 1 & - & - & - & - & - & - & - & - \\
\hline LU_1007 & 1 & 1 & - & 1 & - & 1 & 1 & 1 & 1 & 1 & - & 1 & - & - & - & - & - & - & - & - \\
\hline LU_1011 & 187 & 203 & 173 & 173 & 128 & 155 & 123 & 166 & 163 & 143 & 149 & 89 & - & - & - & - & - & - & - & - \\
\hline LU_2002 & 2 & 2 & 3 & 2 & 3 & 4 & 7 & 3 & 3 & 2 & 3 & 1 & - & - & - & - & - & - & - & - \\
\hline LU_3000 & 3 & 4 & 2 & 2 & - & - & - & 10 & 3 & 3 & 2 & 1 & - & - & - & - & - & - & - & - \\
\hline LU_6401 & 357 & 368 & 407 & 379 & 335 & 389 & 375 & 347 & 362 & 357 & 308 & 254 & - & - & - & - & - & - & - & - \\
\hline MFHUnits & 42 & 40 & 38 & 40 & 47 & 45 & 42 & 34 & 49 & 45 & 39 & 18 & - & - & - & - & - & - & - & - \\
\hline TourUnits & 35 & 12 & 54 & 28 & 35 & 55 & 46 & 25 & 49 & 43 & 45 & 19 & - & - & - & - & - & - & - & - \\
\hline CommSF & 6,976 & 4,412 & 3,231 & 4,396 & - & - & - & 28,036 & 21,558 & 7,323 & 11,024 & 4,531 & - & - & - & - & - & - & - & - \\
\hline
\end{tabular}


Table 7. TahoeTransitionsByYear.txt.

[Y1 through Y20 designate the year of the transition; "ALLOC_DG,” a parcel is allocated to Douglas County; "ALLOC_EL," a parcel is allocated to Eldorado County; "ALLOC_PL," a parcel is allocated to Placer County; "ALLOC_PL_SENSITIVE," a parcel is allocated to the sensitive lots of Placer County;

"ALLOC_WA," a parcel is allocated to Washoe County; "ALLOC_SLT," a parcel is allocated to the city of South Lake Tahoe; "ALLOC_MFH," a parcel is allocated to multiple family housing; "ALLOC_TOUR," a parcel is allocated to tourist accommodation units; "ALLOC_COMM," a parcel is allocated to commercial square footage; "RETIRE_CTC_PRIORITY," a sensitive parcel is allocated to the retirement program of the California Tahoe Conservancy;

"RETIRE_CTC_SECONDARY," a non-sensitive parcel is allocated to the retirement program of the California Tahoe Conservancy;

"RETIRE NEV PRIORITY," a sensitive parcel is allocated to the retirement program of the Nevada State Lands; "RETIRE NEV SECONDARY," a non-

sensitive parcel is allocated to the retirement program of the Nevada State Lands; "RETIRE_TRPA_PRIORITY," a sensitive parcel is allocated to the retirement program of the TRPA; "RETIRE_TRPA_SECONDARY," a non-sensitive parcel is allocated to the retirement program of the TRPA;

"RETIRE USFS PRIORITY," a sensitive parcel is allocated to the retirement program of the U.S. Forest Service; "RETIRE USFS SECONDARY," a nonsensitive parcel is allocated to the retirement program of the U.S. Forest Service]

\begin{tabular}{|c|c|c|c|c|c|c|c|c|c|c|c|c|c|c|c|c|c|c|c|c|}
\hline Desc & $\mathrm{Y} 1$ & Y2 & Y3 & $\mathrm{Y} 4$ & Y5 & Y6 & Y7 & $\mathrm{Y} 8$ & Y9 & $\mathrm{Y} 10$ & Y11 & $\mathrm{Y} 12$ & Y13 & Y14 & $\mathrm{Y} 15$ & Y16 & Y17 & Y18 & Y19 & $\mathrm{Y} 20$ \\
\hline "ALLOC_DG & 12 & 17 & $\overline{14}$ & $\overline{14}$ & 15 & 12 & 2 & - & - & - & - & - & - & - & - & - & - & - & - & - \\
\hline ALLOC_EL & 74 & 67 & 58 & 67 & 45 & 70 & 64 & 87 & 78 & 71 & 77 & 32 & - & - & - & - & - & - & - & - \\
\hline ALLOC_PL & 34 & 51 & 46 & 45 & 38 & 44 & 36 & 50 & 53 & 46 & 44 & 30 & - & - & - & - & - & - & - & - \\
\hline ALLOC_PL_SENSITIVE & - & - & - & - & - & - & - & - & - & - & - & - & - & - & - & - & - & - & - & - \\
\hline ALLOC_WA & 31 & 41 & 24 & 18 & 1 & - & - & - & - & - & - & - & - & - & - & - & - & - & - & - \\
\hline ALLOC_SLT & 36 & 29 & 32 & 29 & 30 & 31 & 21 & 29 & 32 & 27 & 29 & 28 & - & - & - & - & - & - & - & - \\
\hline ALLOC_MFH & 14 & 11 & 13 & 11 & 18 & 12 & 11 & 8 & 11 & 14 & 12 & 5 & - & - & - & - & - & - & - & - \\
\hline ALLOC_TOUR & 2 & 2 & 3 & 2 & 3 & 4 & 7 & 3 & 3 & 2 & 3 & 1 & - & - & - & - & - & - & - & - \\
\hline ALLOC_COMM & 3 & 4 & 2 & 2 & - & - & - & 10 & 3 & 3 & 2 & 1 & - & - & - & - & - & - & - & - \\
\hline RETIRE_CTC_PRIORITY & 59 & 86 & 99 & 73 & 59 & 93 & 88 & 55 & - & - & - & - & - & - & - & - & - & - & - & - \\
\hline RETIRE_CTC_SECONDARY & 79 & 84 & 101 & 79 & 61 & 81 & 93 & 120 & 189 & 171 & 140 & 124 & - & - & - & - & - & - & - & - \\
\hline RETIRE_NEV_PRIORITY & 17 & 16 & 14 & 14 & 18 & 13 & 12 & 4 & - & - & - & - & - & - & - & - & - & - & - & - \\
\hline RETIRE_NEV_SECONDARY & 22 & 17 & 11 & 17 & 18 & 16 & 14 & 3 & - & - & - & - & - & - & - & - & - & - & - & - \\
\hline RETIRE_TRPA_PRIORITY & 17 & 21 & 17 & 20 & 17 & 18 & 17 & 11 & - & - & - & - & - & - & - & - & - & - & - & - \\
\hline RETIRE_TRPA_SECONDARY & 18 & 16 & 21 & 17 & 22 & 17 & 21 & 27 & 41 & 38 & 37 & 29 & - & - & - & - & - & - & - & - \\
\hline RETIRE_USFS_PRIORITY & 73 & 62 & 73 & 83 & 74 & 81 & 57 & 42 & - & - & - & - & - & - & - & - & - & - & - & - \\
\hline RETIRE_USFS_SECONDARY & 73 & 69 & 73 & 77 & 69 & 71 & 75 & 87 & 132 & 149 & 132 & 102 & - & - & - & - & - & - & - & - \\
\hline
\end{tabular}


Table 8. TahoeTransCandidatesByYear.txt.

[Y1 through Y20 designate the year of the transition; "ALLOC_DG,” a parcel is allocated to Douglas County; "ALLOC_EL," a parcel is allocated to Eldorado County; "ALLOC_PL," a parcel is allocated to Placer County; "ALLOC_PL_SENSITIVE," a parcel is allocated to the sensitive lots of Placer County;

"ALLOC_WA," a parcel is allocated to Washoe County; "ALLOC_SLT," a parcel is allocated to the city of South Lake Tahoe; "ALLOC_MFH," a parcel is allocated to multiple family housing; "ALLOC_TOUR," a parcel is allocated to tourist accommodation units; "ALLOC_COMM," a parcel is allocated to commercial square footage; "RETIRE_CTC_PRIORITY," a sensitive parcel is allocated to the retirement program of the California Tahoe Conservancy;

"RETIRE_CTC_SECONDARY," a non-sensitive parcel is allocated to the retirement program of the California Tahoe Conservancy;

"RETIRE NEV PRIORITY," a sensitive parcel is allocated to the retirement program of the Nevada State Lands; "RETIRE NEV SECONDARY," a non-

sensitive parcel is allocated to the retirement program of the Nevada State Lands; "RETIRE_TRPA_PRIORITY," a sensitive parcel is allocated to the retirement program of the TRPA; "RETIRE_TRPA_SECONDARY," a non-sensitive parcel is allocated to the retirement program of the TRPA;

"RETIRE USFS PRIORITY," a sensitive parcel is allocated to the retirement program of the U.S. Forest Service; "RETIRE USFS SECONDARY," a nonsensitive parcel is allocated to the retirement program of the U.S. Forest Service]

\begin{tabular}{|c|c|c|c|c|c|c|c|c|c|c|c|c|c|c|c|c|c|c|c|c|c|}
\hline Desc & YO & Y1 & $\mathrm{Y} 2$ & $\mathrm{Y} 3$ & $\mathrm{Y} 4$ & Y5 & Y6 & Y7 & $\mathrm{Y} 8$ & $\mathrm{Y9}$ & $\mathrm{Y} 10$ & Y11 & Y12 & Y13 & Y14 & Y15 & Y16 & Y17 & $\mathrm{Y} 18$ & Y19 & $\mathrm{Y} 20$ \\
\hline "ALLOC_DG & 150 & 123 & $\overline{899}$ & 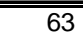 & 39 & 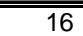 & $\overline{2} 2$ & - & - & - & - & - & - & - & - & - & - & - & - & - & - \\
\hline ALLOC_El & 1,823 & 1,649 & 1,475 & 1,305 & 1,147 & 1,019 & 849 & 697 & 533 & 341 & 180 & 45 & - & - & - & - & - & - & - & - & - \\
\hline ALLOC_PL & 1,130 & 1,109 & 1,014 & 913 & 813 & 731 & 625 & 535 & 421 & 295 & 168 & 66 & - & - & - & - & - & - & - & - & - \\
\hline ALLOC_PL_SENSTTVE & 60 & - & - & - & - & - & - & - & - & - & - & - & - & - & - & - & - & - & - & - & - \\
\hline ALLOC_WA & 155 & 105 & 52 & 22 & 1 & - & - & - & - & - & - & - & - & - & - & - & - & - & - & - & - \\
\hline ALLOC_SLT & 1,268 & 1,177 & 1,089 & 991 & 888 & 801 & 707 & 600 & 487 & 362 & 234 & 107 & - & - & - & - & - & - & - & - & - \\
\hline ALLOC_MFH & 797 & 716 & 638 & 561 & 498 & 431 & 358 & 300 & 236 & 164 & 86 & 29 & - & - & - & - & - & - & - & - & - \\
\hline ALLOC_TOUR & 98 & 91 & 83 & 70 & 62 & 55 & 47 & 34 & 26 & 18 & 12 & 4 & - & - & - & - & - & - & - & - & - \\
\hline$\overline{\text { ALLOC_COMM }}$ & 286 & 263 & 241 & 222 & 203 & 184 & 166 & 146 & 121 & 91 & 61 & 31 & - & - & - & - & - & - & - & - & - \\
\hline RETIRE_CTC_PRIORITY & 1,299 & 1,145 & 978 & 791 & 616 & 459 & 266 & 106 & - & - & - & - & - & - & - & - & - & - & - & - & - \\
\hline RETIRE_CTC_SECONDARY & 4,463 & 4,155 & 3,846 & 3,521 & 3,209 & 2,939 & 2,621 & 2,299 & 1,880 & 1,340 & 822 & 349 & - & - & - & - & - & - & - & - & - \\
\hline RETIRE_NEV_PRIORITY & 260 & 212 & 154 & 110 & 75 & 47 & 24 & 6 & - & - & - & - & - & - & - & - & - & - & - & - & - \\
\hline RETIRE_NEV_SECONDARY & 259 & 208 & 155 & 115 & 70 & 43 & 18 & 3 & - & - & - & - & - & - & - & - & - & - & - & - & - \\
\hline RETIRE_TRPA_PRIORITY & 1,559 & 1,357 & 1,131 & 901 & 691 & 505 & 290 & 111 & - & - & - & - & - & - & - & - & - & - & - & - & - \\
\hline RETIRE_TRPA_SECONDARY & 4,722 & 4,362 & 4,001 & 3,635 & 3,279 & 2,982 & 2,639 & 2,302 & 1,880 & 1,340 & 822 & 349 & - & - & - & - & - & - & - & - & - \\
\hline RETIRE_USFS_PRIORITY & 1,559 & 1,357 & 1,131 & 901 & 691 & 505 & 290 & 111 & - & - & - & - & - & - & - & - & - & - & - & - & - \\
\hline RETIRE_USFS_SECONDARY & 4,722 & 4,362 & 4,001 & 3,635 & 3,279 & 2,982 & 2,639 & 2,302 & 1,880 & 1,340 & 822 & 349 & - & - & - & - & - & - & - & - & - \\
\hline
\end{tabular}


Table 9. TahoeCategoryTotals.txt.

["Category", a particular combination of fates for a given parcel; "Count", number of times that category and combination of fates occurs; "LU_XXXX", a different formulation of the TRPA land use codes and the values are the percent of the time the model designates this particular land use]

\begin{tabular}{|r|r|r|r|r|r|r|r|}
\hline Category & rount & LU_0001 & LU_MFH & LU_1011 & LU_2002 & LU_3000 & LU_6401 \\
\hline \hline 1 & 1435 & $0.0 \%$ & $0.0 \%$ & $0.0 \%$ & $0.0 \%$ & $0.0 \%$ & $100.0 \%$ \\
\hline 4 & 1228 & $0.0 \%$ & $0.0 \%$ & $50.3 \%$ & $0.0 \%$ & $0.0 \%$ & $49.7 \%$ \\
\hline 6 & 949 & $0.0 \%$ & $0.0 \%$ & $46.5 \%$ & $0.0 \%$ & $0.0 \%$ & $53.5 \%$ \\
\hline 2 & 855 & $0.0 \%$ & $0.0 \%$ & $31.8 \%$ & $0.0 \%$ & $0.0 \%$ & $68.2 \%$ \\
\hline 5 & 320 & $0.0 \%$ & $0.0 \%$ & $16.4 \%$ & $0.0 \%$ & $0.0 \%$ & $83.6 \%$ \\
\hline 12 & 260 & $0.0 \%$ & $23.8 \%$ & $23.3 \%$ & $0.0 \%$ & $0.0 \%$ & $52.9 \%$ \\
\hline 14 & 252 & $0.0 \%$ & $16.5 \%$ & $44.4 \%$ & $0.0 \%$ & $0.0 \%$ & $39.1 \%$ \\
\hline 16 & 149 & $0.0 \%$ & $15.1 \%$ & $36.9 \%$ & $0.0 \%$ & $0.0 \%$ & $48.0 \%$ \\
\hline 3 & 118 & $0.0 \%$ & $0.0 \%$ & $12.7 \%$ & $0.0 \%$ & $0.0 \%$ & $87.3 \%$ \\
\hline 8 & 94 & $0.0 \%$ & $0.0 \%$ & $58.5 \%$ & $0.0 \%$ & $0.0 \%$ & $41.5 \%$ \\
\hline 32 & 74 & $0.0 \%$ & $0.0 \%$ & $0.0 \%$ & $0.0 \%$ & $15.5 \%$ & $84.5 \%$ \\
\hline 10 & 64 & $0.0 \%$ & $0.0 \%$ & $82.0 \%$ & $0.0 \%$ & $0.0 \%$ & $18.0 \%$ \\
\hline 7 & 59 & $0.0 \%$ & $0.0 \%$ & $14.4 \%$ & $0.0 \%$ & $0.0 \%$ & $85.6 \%$ \\
\hline 9 & 53 & $0.0 \%$ & $0.0 \%$ & $51.9 \%$ & $0.0 \%$ & $0.0 \%$ & $48.1 \%$ \\
\hline 11 & 49 & $0.0 \%$ & $0.0 \%$ & $72.4 \%$ & $0.0 \%$ & $0.0 \%$ & $27.6 \%$ \\
\hline 30 & 41 & $0.0 \%$ & $0.0 \%$ & $0.0 \%$ & $0.0 \%$ & $13.4 \%$ & $86.6 \%$ \\
\hline 31 & 31 & $0.0 \%$ & $0.0 \%$ & $0.0 \%$ & $0.0 \%$ & $14.5 \%$ & $85.5 \%$ \\
\hline 13 & 28 & $0.0 \%$ & $16.1 \%$ & $5.4 \%$ & $0.0 \%$ & $0.0 \%$ & $78.6 \%$ \\
\hline 0 & 25 & $0.0 \%$ & $2.0 \%$ & $14.0 \%$ & $14.0 \%$ & $0.0 \%$ & $70.0 \%$ \\
\hline 27 & 25 & $0.0 \%$ & $0.0 \%$ & $0.0 \%$ & $40.0 \%$ & $10.0 \%$ & $50.0 \%$ \\
\hline 15 & 23 & $0.0 \%$ & $4.3 \%$ & $13.0 \%$ & $0.0 \%$ & $0.0 \%$ & $82.6 \%$ \\
\hline 18 & 22 & $0.0 \%$ & $4.5 \%$ & $61.4 \%$ & $0.0 \%$ & $0.0 \%$ & $34.1 \%$ \\
\hline 28 & 22 & $0.0 \%$ & $0.0 \%$ & $0.0 \%$ & $43.2 \%$ & $6.8 \%$ & $50.0 \%$ \\
\hline 20 & 20 & $0.0 \%$ & $0.0 \%$ & $25.0 \%$ & $25.0 \%$ & $10.0 \%$ & $40.0 \%$ \\
\hline 17 & 14 & $0.0 \%$ & $3.6 \%$ & $64.3 \%$ & $0.0 \%$ & $0.0 \%$ & $32.1 \%$ \\
\hline 19 & 12 & $0.0 \%$ & $8.3 \%$ & $33.3 \%$ & $0.0 \%$ & $0.0 \%$ & $58.3 \%$ \\
\hline 24 & 12 & $0.0 \%$ & $12.5 \%$ & $0.0 \%$ & $0.0 \%$ & $8.3 \%$ & $79.2 \%$ \\
\hline 22 & 9 & $0.0 \%$ & $11.1 \%$ & $0.0 \%$ & $0.0 \%$ & $0.0 \%$ & $88.9 \%$ \\
\hline 26 & 7 & $0.0 \%$ & $0.0 \%$ & $0.0 \%$ & $35.7 \%$ & $0.0 \%$ & $64.3 \%$ \\
\hline 29 & 7 & $0.0 \%$ & $0.0 \%$ & $0.0 \%$ & $7.1 \%$ & $0.0 \%$ & $92.9 \%$ \\
\hline 33 & 7 & $0.0 \%$ & $0.0 \%$ & $0.0 \%$ & $0.0 \%$ & $0.0 \%$ & $100.0 \%$ \\
\hline 21 & 6 & $0.0 \%$ & $0.0 \%$ & $66.7 \%$ & $0.0 \%$ & $0.0 \%$ & $33.3 \%$ \\
\hline 25 & 4 & $0.0 \%$ & $0.0 \%$ & $0.0 \%$ & $0.0 \%$ & $0.0 \%$ & $100.0 \%$ \\
\hline 34 & 4 & $0.0 \%$ & $0.0 \%$ & $0.0 \%$ & $0.0 \%$ & $0.0 \%$ & $100.0 \%$ \\
\hline 23 & 3 & $0.0 \%$ & $0.0 \%$ & $0.0 \%$ & $0.0 \%$ & $0.0 \%$ & $100.0 \%$ \\
\hline
\end{tabular}




\section{Appendixes}

\section{Appendix 1: Tahoe Regional Planning Agency's Parcel Land Use Codes}

Bolded codes are used in the LUSM.

(Updated 10-8-98)

$$
\underline{0-999}
$$

\section{MISCELLANEOUS}

N

\begin{tabular}{ll}
0 & Unknown \\
$\mathbf{1}$ & $\begin{array}{l}\text { Vacant (private) } \\
\text { Streets/Highways }\end{array}$ \\
99 & RESIDENTIAL \\
$\mathbf{1 0 0 0}$ & Domestic animal raising \\
\hline 1001 & Employee housing \\
1002 & Mobile home dwelling \\
1003 & * Mobile Home Park \\
1004 & Multiple family dwelling (2-4 units) \\
$\mathbf{1 0 0 5}$ & Multiple family dwelling (5-10 units) \\
$\mathbf{1 0 0 6}$ & Multiple family dwelling (10+ units) \\
$\mathbf{1 0 0 7}$ & Multi-person dwelling \\
1008 & Nursing and personal care \\
1009 & Residential care \\
1010 & Single family dwelling (Existing) \\
$\mathbf{1 0 1 1}$ & Single family dwelling (New) \\
1012 & Summer home \\
1013 & Condominium \\
1014 & Condominium Common Area \\
1015 & Accessory use to a Single Family Dwelling \\
1016 & Abandoned Residential Structure \\
1017 & TOURIST ACCOMMODATION \\
&
\end{tabular}

\begin{tabular}{ll}
2001 & $\begin{array}{l}\text { Bed and breakfast facilities } \\
\text { Hotel, motel, and other transient dwelling units } \\
\text { Time sharing (hotel/motel design) } \\
\text { Time sharing (residential design) } \\
2004\end{array}$ \\
$\mathbf{3 0 0 0}$ & COMMERCIAL \\
\hline 3100 & A. Retail \\
3101 & Auto, mobile home and vehicle dealers \\
3102 & Building materials and hardware \\
3103 & General merchandise stores \\
3104 & Mail order and vending \\
3105 & Nursery \\
3106 & Outdoor retail sales \\
3107 & Eating and drinking places \\
3108 & Food and beverage retail sales \\
3109 & Furniture, home furnishings and equipment \\
3110 & Service Stations \\
& B. Entertainment \\
3200 & Amusements and recreation services \\
3201 & Gaming-nonrestricted (Nevada only) \\
3202 & Privately owned assembly and entertainment \\
3203 & Outdoor amusements \\
3204 & C. Services \\
3300 &
\end{tabular}


Animal husbandry services

Auto repair and service

Broadcasting studios

Business support services

Contract construction services

Financial services

Health care services

Laundries and dry cleaning plant

Personal services

Professional offices

Repair services

Sales lots

Schools - business and vocational

Secondary storage

\section{Light Industrial}

Batch plants

Food and kindred products

Fuel and ice dealers

Industrial services

Printing and publishing

Recycling and scrap

Small scale manufacturing

\section{E. Wholesale/Storage}

Storage yards

Vehicle and freight terminals

Vehicle storage and parking

Warehousing

Wholesale and distribution

* Abandoned Commercial Structure

\section{PUBLIC SERVICE}

$\begin{array}{ll}4100 & \text { A. General } \\ 4101 & \text { Airfields, landing strips, heliports (New non-emergency sites } \\ \text { prohibited) } & \text { Cemeteries } \\ 4102 & \text { Churches } \\ 4103 & \text { Collection stations } \\ 4104 & \text { Cultural facilities } \\ 4105 & \text { Day care centers/pre-schools } \\ 4106 & \end{array}$

$\begin{array}{ll}4107 & \text { Government offices } \\ 4108 & \text { Hospitals } \\ 4109 & \text { Local assembly and entertainment } \\ 4110 & \text { Local post office } \\ 4111 & \text { Local public health and safety facilities } \\ 4112 & \text { Power generating } \\ 4113 & \text { Public owned assembly and entertainment } \\ 4114 & \text { Public utility centers } \\ 4115 & \text { Regional public health and safety facilities } \\ 4116 & \text { Schools - college } \\ 4117 & \text { Schools - Kindergarten through secondary } \\ 4118 & \text { Social service organizations } \\ & \\ 4200 & \text { B. Linear Public Facilities } \\ 4201 & \text { Pipelines and power transmission } \\ 4202 & \text { Transit stations and terminals } \\ 4203 & \text { Transportation routes } \\ 4204 & \text { Transmission and receiving facilities }\end{array}$

\section{RECREATION}

\section{$5001 \quad$ Beach recreation}

$5002 \quad$ Boat launching facilities

$5003 \quad$ Cross country ski courses

$5004 \quad$ Day use areas

$5005 \quad$ Developed campgrounds

$5006 \quad$ Downhill ski facilities

$5007 \quad$ Golf courses

$5008 \quad$ Group facilities

$5009 \quad$ Marinas

$5010 \quad$ Off road vehicle courses

$5011 \quad$ Outdoor recreation concessions

$5012 \quad$ Participant sports facilities

$5013 \quad$ Recreation centers

$5014 \quad$ Recreations vehicle parks

$5015 \quad$ Riding and hiking trails

$5016 \quad$ Rural Sports

$5017 \quad$ Snowmobile courses

$5018 \quad$ Sport Assembly

$5019 \quad$ Undeveloped campgrounds

$5020 \quad$ Visitor information center

6000

RESOURCE MANAGEMENT 


$$
\underline{6100}
$$

A. Timber Management

\begin{tabular}{ll}
6101 & Reforestation \\
6102 & Regeneration harvest \\
6103 & Sanitation salvage cut \\
6104 & Selection cut \\
6105 & Special cut \\
6106 & Thinning \\
6107 & Timber stand improvement \\
6108 & Tree farms \\
& \\
6200 & B. Wildlife and Fishes \\
\hline
\end{tabular}

$6201 \quad$ Early successional vegetation management

$6202 \quad$ Non-structural fish habitat management

$6203 \quad$ Non-structural wildlife habitat management

$6204 \quad$ Structural fish habitat management

6205 Structural wildlife habitat management

$\underline{6300 \quad \text { C. Range }}$

\begin{tabular}{ll}
6301 & Farm/Ranch structures \\
6302 & Grazing \\
6303 & Range pasture management \\
6304 & Range improvement \\
6400 & D. Open Space \\
\hline
\end{tabular}

\begin{tabular}{|c|c|}
\hline 6401 & Allowed in all areas of the region \\
\hline 6500 & E. Vegetation Protection \\
\hline 6501 & Fire detection and suppression \\
\hline 6502 & Fuels treatment/management \\
\hline 6503 & Insect and disease suppression \\
\hline 6504 & Prescribed fire/burning management \\
\hline 6505 & Sensitive plant management \\
\hline 6506 & Uncommon plant community management \\
\hline$\underline{6600}$ & F. Watershed Improvements \\
\hline 6601 & Erosion control \\
\hline 6602 & Runoff control \\
\hline 6603 & Stream environment zone restoration \\
\hline
\end{tabular}

7010

7020

7030

7040

7050

7060

7070

7080

7090

7100

7110

7120

7130

7140

7150

Safety and navigational facilities

Salvage operations

Seaplane operations

Tour boat operations

Water borne transit

Water intake lines

Beach recreation

Boat launching facilities

Construction equipment storage

Marinas

Water oriented outdoor recreation concessions

Commercial boating

Parasailing

Fish Habitat Restoration

Scientific Study

Shorezone Accessories (4th digit in project code)

\section{Boat ramp}

Breakwaters or jetties

Buoys

Fences

Floating docks and platforms

Piers

Shoreline protective structures

Water intake lines

Beach raking 


\section{EIP PROJECT CODES}

9000

9010

9011

9012

9013

9014

9080

9081

9090

9091

\section{WATER QUALITY}

Research/Evaluation Project

9120 SEZ Restoration Project

$9130 \quad$ SEZ Creation Project

9140 Erosion Control/WQ Treatment

9150 Soil Conservation Project

$9180 \quad$ Water Quality Programs

$9181 \quad$ Research and Evaluation (TRPA)

$9182 \quad$ SEZ Research

$9190 \quad$ Water Quality Regulations

\section{$\underline{9200}$}

\section{AQ/TRANSPORTATION}

$\begin{array}{ll}9210 & \text { Transit Project } \\ 9220 & \text { Highway Project } \\ 9230 & \text { Airport Project } \\ 9240 & \text { Bikeway Project } \\ 9250 & \text { Pedestrian Project } \\ 9260 & \text { Multi-Purpose Project } \\ 9270 & \text { Parking Project } \\ 9280 & \text { AQ/Transportation Program } \\ 9281 & \text { Research and Evaluation (TRPA) } \\ 9290 & \text { AQ/Transportation Regulation } \\ & \\ \mathbf{9 3 0 0} & \text { NOISE } \\ 9310 & \\ 9380 & \text { Noise Reduction Project } \\ 9381 & \text { Noise Reduction Program } \\ 9390 & \text { Research and Evaluation (TRPA) } \\ & \text { Noise Reduction Regulations }\end{array}$

PAOT Projects

Overnight

Day Use

Winter

Marinas/Ramps

Non PAOT Projects

Recreation Land Acquisition

Recreation Program

Research and Evaluation (TRPA)

Recreation Regulation

\section{$\underline{9500 \quad \text { FISHERY }}$}

$\begin{array}{ll}9510 & \text { Stream } \\ 9520 & \text { Lake } \\ 9580 & \text { Fishery Program } \\ 9581 & \text { Research and Evaluation - In Stream } \\ 9582 & \text { Research and Evaluation - In Lake } \\ 9590 & \text { Fishery Regulation } \\ 9591 & \text { In-Stream Fisheries } \\ 9592 & \text { In-Lake Fisheries } \\ & \\ \mathbf{9 6 0 0} & \text { WILDLIFE }\end{array}$

$9610 \quad$ Wildlife Enhancement Projects

$9680 \quad$ Wildlife Programs

$9681 \quad$ Research and Evaluation (TRPA)

$9690 \quad$ Wildlife Regulation

$9700 \quad$ SCENIC

$9710 \quad$ Scenic Restoration Project

$9720 \quad$ Undergrounding

$9780 \quad$ Scenic Programs

$9781 \quad$ Research and Evaluation (TRPA)

$9790 \quad$ Scenic Regulation

\section{$\underline{9800 \quad \text { VEGETATION }}$}

$9810 \quad$ Forest Project

$9880 \quad$ Vegetation Program 
Research and Evaluation (TRPA)

$9890 \quad$ Vegetation Regulation

9900

OTHER

$9910 \quad$ Health and Safety 


\section{Appendix 2: Assessor's 2008 Parcel Map Data Summary}

[Level I of the table represents the first major category of land uses as defined in appendix 1 (e.g. 1000), level II represents the second category (e.g. 3100), and level III represents the most specific hierarchical level of the land use categories (e.g. 3105)]

\begin{tabular}{|c|c|c|c|c|c|c|c|c|c|c|c|c|}
\hline \multicolumn{2}{|c|}{ Level I } & \multirow{2}{*}{\multicolumn{2}{|c|}{ Level II }} & \multirow[b]{3}{*}{ Description } & \multirow{2}{*}{\multicolumn{2}{|c|}{ Level III }} & & $\begin{array}{c}\text { Level II } \\
\text { Total } \\
\end{array}$ & $\begin{array}{c}\text { Level III } \\
\text { Total } \\
\end{array}$ \\
\hline \multirow[b]{2}{*}{ Description } & \multirow[b]{2}{*}{ Count } & & & & & & & & Area i & Acres & & \\
\hline & & Description & Count & & Count & Landuse & Min Area & Med Area & Max Area & Total Area & \multirow[b]{2}{*}{6,707} & \multirow[b]{2}{*}{6,707} \\
\hline Unknown (null) & 210 & & 210 & Unknown (or null) & 210 & 0 & 0.0 & 0.1 & 4,182 & 6,707 & & \\
\hline Vacant & 6,326 & & 6,326 & Vacant & 6,326 & 1 & 0.0 & 0.2 & 468 & 6,801 & 6,801 & 6,801 \\
\hline \multirow[t]{13}{*}{ Residential } & 41,219 & & 41,219 & Domestic Animal Raising & 1 & 1001 & 24.3 & 24.3 & 24 & 24 & 13,790 & 13,790 \\
\hline & & & & Mobile Home Dwelling & 747 & 1003 & 0.0 & 0.0 & 1 & 30 & & \\
\hline & & & & Mobile Home Park & 34 & 1004 & 0.1 & 0.9 & 23 & 82 & & \\
\hline & & & & Multiple Family Dwelling (2-4 units) & 1,697 & 1005 & 0.0 & 0.1 & 41 & 592 & & \\
\hline & & & & Multiple Family Dwelling (5-10 units) & 196 & 1006 & 0.1 & 0.2 & 20 & 79 & & \\
\hline & & & & Multiple Family Dwelling (10+ units) & 82 & 1007 & 0.0 & 0.5 & 7 & 86 & & \\
\hline & & & & Nursing and Personal Care & 1 & 1009 & 1.5 & 1.5 & 1 & 1 & & \\
\hline & & & & Residential Care & 1 & 1010 & 1.3 & 1.3 & 1 & 1 & & \\
\hline & & & & Single Family Dwelling (existing) & 28,927 & 1011 & 0.0 & 0.2 & 596 & 10,825 & & \\
\hline & & & & Summer Home & 518 & 1013 & 0.1 & 0.3 & 134 & 378 & & \\
\hline & & & & Condominium & 7,988 & 1014 & 0.0 & 0.0 & 9 & 218 & & \\
\hline & & & & Condominium Common Area & 725 & 1015 & 0.0 & 0.5 & 87 & 1,372 & & \\
\hline & & & & Accessory use to a Single Family Dwelling & 302 & 1016 & 0.0 & 0.2 & 4 & 100 & & \\
\hline \multirow[t]{4}{*}{ Tourist } & 427 & & 427 & Bed and Breakfast Facilities & 3 & 2001 & 0.3 & 0.3 & 1 & 2 & 389 & 389 \\
\hline & & & & Hotel, Motel, and other Transient Dwelling Units & 256 & 2002 & 0.1 & 0.5 & 22 & 320 & & \\
\hline & & & & Time Sharing (Hotel/Motel Design) & 20 & 2003 & 0.0 & 0.6 & 6 & 25 & & \\
\hline & & & & Time Sharing (Residential Design) & 148 & 2004 & 0.0 & 0.0 & 24 & 42 & & \\
\hline \multirow[t]{11}{*}{ Commercial } & 1,279 & Retail & 430 & $\mathrm{n} / \mathrm{a}$ & 1 & 3100 & 0.2 & 0.2 & 0 & 0 & 340 & 971 \\
\hline & & & & Auto, Mobile Home and Vehicle Dealers & 8 & 3101 & 0.2 & 0.5 & 3 & 8 & & \\
\hline & & & & Building Materials and Hardware & 17 & 3102 & 0.2 & 0.5 & 4 & 18 & & \\
\hline & & & & General Merchandise Stores & 197 & 3103 & 0.0 & 0.3 & 18 & 160 & & \\
\hline & & & & Mail Order and Vending & 6 & 3105 & 0.3 & 0.4 & 1 & 3 & & \\
\hline & & & & Nursery & 7 & 3106 & 0.1 & 0.3 & 1 & 2 & & \\
\hline & & & & Outdoor Retail Sales & 112 & 3107 & 0.0 & 0.4 & 5 & 73 & & \\
\hline & & & & Eating and Drinking Places & 25 & 3108 & 0.0 & 0.5 & 14 & 33 & & \\
\hline & & & & Food and Beverage Retail Sales & 9 & 3109 & 0.1 & 0.3 & 1 & 3 & & \\
\hline & & & & Furniture, Home Furnishings and Equipment & 35 & 3110 & 0.1 & 0.5 & 1 & 17 & & \\
\hline & & & & Service Stations & 13 & 3111 & 0.1 & 0.8 & 8 & 21 & & \\
\hline
\end{tabular}




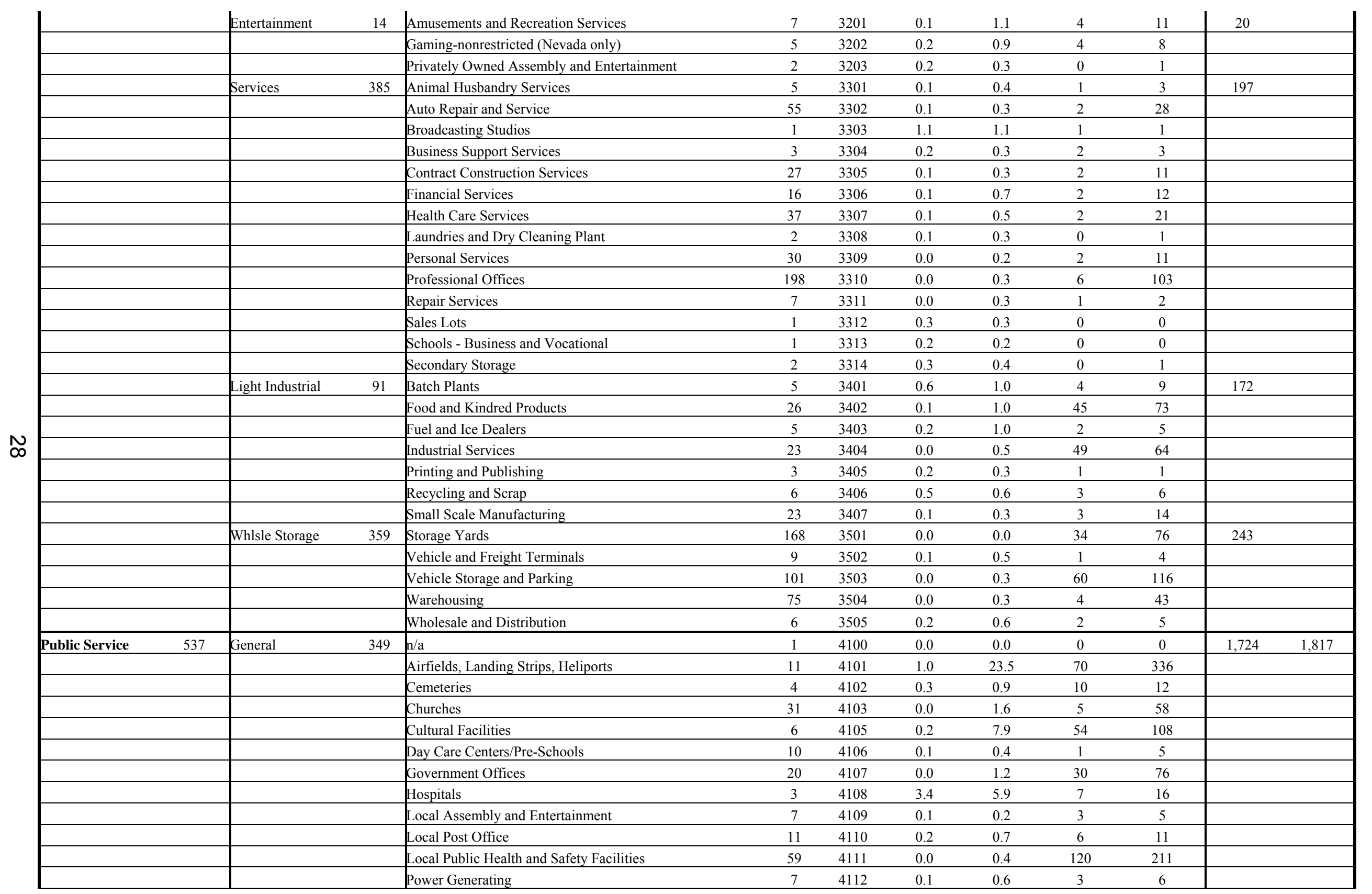




\begin{tabular}{|c|c|c|c|c|c|c|c|c|c|c|c|c|}
\hline & & & & Public Owned Assembly and Entertainment & 1 & 4113 & 13.4 & 13.4 & 13 & 13 & & \\
\hline & & & & Public Utility Centers & 115 & 4114 & 0.0 & 0.2 & 87 & 257 & & \\
\hline & & & & Regional public health and Safety Facilities & 18 & 4115 & 0.1 & 0.7 & 33 & 66 & & \\
\hline & & & & Schools - College & 7 & 4116 & 0.4 & 1.6 & 113 & 132 & & \\
\hline & & & & Schools - Kindergarten through Secondary & 31 & 4117 & 0.2 & 5.0 & 80 & 409 & & \\
\hline & & & & Social Services Organizations & 7 & 4118 & 0.0 & 0.2 & 2 & 3 & & \\
\hline & & Linear Facilities & 188 & Pipelines and Power Transmission & 3 & 4201 & 0.0 & 0.3 & 0 & 1 & 93 & \\
\hline & & & & Transit Stations and Terminals & 3 & 4202 & 0.6 & 0.8 & 11 & 12 & & \\
\hline & & & & Transportation Routes & 176 & 4203 & 0.0 & 0.2 & 9 & 76 & & \\
\hline & & & & Transmission and Receiving Facilities & 6 & 4204 & 0.0 & 0.5 & 2 & 4 & & \\
\hline \multirow[t]{15}{*}{ Recreation } & 621 & & 621 & Beach Recreation & 71 & 5001 & 0.0 & 1.2 & 226 & 389 & 19,693 & 19,693 \\
\hline & & & & Boat Launching Facilities & 8 & 5002 & 0.4 & 2.3 & 11 & 26 & & \\
\hline & & & & Day Use Areas & 150 & 5004 & 0.0 & 7.0 & 3,765 & 12,403 & & \\
\hline & & & & Developed Campgrounds & 16 & 5005 & 5.1 & 36.0 & 307 & 1,172 & & \\
\hline & & & & Downhill Ski Facilities & 91 & 5006 & 0.1 & 10.5 & 821 & 4,262 & & \\
\hline & & & & Golf Courses & 44 & 5007 & 0.0 & 4.7 & 224 & 1,013 & & \\
\hline & & & & Group Facilities & 9 & 5008 & 0.7 & 9.6 & 33 & 110 & & \\
\hline & & & & Marinas & 177 & 5009 & 0.0 & 0.0 & 19 & 66 & & \\
\hline & & & & Outdoor Recreation Concessions & 6 & 5011 & 0.2 & 1.0 & 6 & 11 & & \\
\hline & & & & Participant Sports Facilities & 9 & 5012 & 0.2 & 3.5 & 30 & 87 & & \\
\hline & & & & Recreation Centers & 12 & 5013 & 0.9 & 1.5 & 18 & 52 & & \\
\hline & & & & Riding and Hiking Trails & 22 & 5015 & 0.1 & 1.9 & 23 & 96 & & \\
\hline & & & & Rural Sports & 1 & 5016 & 0.7 & 0.7 & 1 & 1 & & \\
\hline & & & & Undeveloped Campgrounds & 1 & 5019 & 4.6 & 4.6 & 5 & 5 & & \\
\hline & & & & Visitor Information Centers & 4 & 5020 & 0.0 & 0.4 & 1 & 2 & & \\
\hline \multirow[t]{2}{*}{ Resource Mgmt } & 9,757 & Open Space & 9,757 & Retired & 9,757 & 6401 & 0.0 & 0.3 & 3,747 & 164,385 & 164,385 & 164,385 \\
\hline & 60,376 & & 60,376 & & 60,376 & & & & & 214,552 & 214,552 & 214,552 \\
\hline
\end{tabular}




\section{Appendix 3: Samples of Required Databases for the Land Use Simulation Model}

Below are samples of records and their attributes of the two primary datasets used in

\section{PostGreSQL.}

Table 1. Parcel database with Plan Area Statement ID linkage.

\begin{tabular}{|l|l|l|l|l|l|l|}
\hline $\begin{array}{c}\text { Assessor's Parcel } \\
\text { Number }\end{array}$ & $\begin{array}{c}\text { Plan Area } \\
\text { Statement ID }\end{array}$ & Jurisdiction & Area (ac) & $\begin{array}{c}\text { IPES } \\
\text { Score }\end{array}$ & $\begin{array}{c}\text { Bailey } \\
\text { Score }\end{array}$ & $\begin{array}{c}\text { South Lake } \\
\text { Tahoe }\end{array}$ \\
\hline $010-170-01$ & 1520 & EL & 49.475 & 0 & 4 & FALSE \\
\hline $010-170-02$ & 1520 & EL & 160.659 & 0 & 4 & FALSE \\
\hline $014-231-05$ & 1540 & EL & 0.263 & 826 & 5 & FALSE \\
\hline $014-231-09$ & 1540 & EL & 0.323 & 874 & 5 & FALSE \\
\hline $014-232-01$ & 1540 & EL & 0.230 & 836 & 5 & FALSE \\
\hline $014-232-03$ & 1540 & EL & 0.203 & 814 & 5 & FALSE \\
\hline $014-232-09$ & 1540 & EL & 0.276 & 814 & 5 & FALSE \\
\hline $014-234-02$ & 1540 & EL & 0.246 & 846 & 5 & FALSE \\
\hline $014-234-11$ & 1540 & EL & 0.246 & 846 & 5 & FALSE \\
\hline $014-235-06$ & 1540 & EL & 0.276 & 836 & $1 \mathrm{~b}$ & FALSE \\
\hline $014-236-05$ & 1540 & EL & 0.230 & 826 & 5 & FALSE \\
\hline $014-236-08$ & 1540 & EL & 0.230 & 804 & 5 & FALSE \\
\hline $014-236-12$ & 1540 & EL & 0.230 & 811 & 5 & FALSE \\
\hline $014-237-03$ & 1540 & EL & 0.230 & 820 & 5 & FALSE \\
\hline $014-237-04$ & 1540 & EL & 0.230 & 820 & 5 & FALSE \\
\hline $014-238-12$ & 1540 & EL & 0.246 & 836 & 5 & FALSE \\
\hline $014-241-07$ & 1540 & EL & 0.230 & 830 & $1 \mathrm{~b}$ & FALSE \\
\hline $014-242-06$ & 1540 & EL & 0.229 & 0 & $1 \mathrm{~b}$ & FALSE \\
\hline $014-243-07$ & 1540 & EL & 0.230 & 440 & 5 & FALSE \\
\hline $014-244-06$ & 1540 & EL & 0.246 & 805 & 5 & FALSE \\
\hline $014-244-11$ & 1540 & EL & 0.246 & 783 & 5 & FALSE \\
\hline $014-247-04$ & 1540 & EL & 1.758 & 0 & 4 & FALSE \\
\hline $014-262-03$ & 1540 & EL & 0.232 & 819 & 5 & FALSE \\
\hline $014-262-10$ & 1540 & EL & 0.244 & 768 & 5 & FALSE \\
\hline $014-262-12$ & 1540 & EL & 0.232 & 807 & 5 & FALSE \\
\hline & & & & & & \\
\hline & & & & & \\
\hline
\end{tabular}


Table 2. Plan Area Statement database

\begin{tabular}{|c|c|c|c|c|c|c|c|}
\hline $\begin{array}{c}\text { Plan Area } \\
\text { Statement ID }\end{array}$ & $\begin{array}{c}\text { Plan Area } \\
\text { Statement Name }\end{array}$ & \begin{tabular}{|c|} 
Multiple \\
Family \\
Dwelling
\end{tabular} & \begin{tabular}{|c|} 
Single \\
Family \\
Dwelling
\end{tabular} & $\begin{array}{c}\text { Tourist } \\
\text { Accommodation }\end{array}$ & $\begin{array}{c}\text { Commercial } \\
\text { Uses }\end{array}$ & $\begin{array}{c}\text { Multiple Family } \\
\text { Dwelling Density } \\
\text { (units/ac) }\end{array}$ & $\begin{array}{c}\text { Tourist } \\
\text { Accommodation } \\
\text { Density (units/ac) }\end{array}$ \\
\hline 001A1 & TAHOE CITY & $\mathrm{N}$ & $\mathrm{N}$ & $\mathrm{A}$ & A & 0 & 40 \\
\hline $001 \mathrm{~A} 2$ & TAHOE CITY & $\mathrm{N}$ & $\mathrm{N}$ & $\mathrm{N}$ & A & 0 & 0 \\
\hline $001 \mathrm{~A} 3$ & TAHOE CITY & $\mathrm{N}$ & $\mathrm{N}$ & $\mathrm{N}$ & $\mathrm{S}$ & 0 & 0 \\
\hline \begin{tabular}{|l|}
$001 \mathrm{~A} 4$ \\
\end{tabular} & TAHOE CITY & $\mathrm{A}$ & $\mathrm{A}$ & $\mathrm{A}$ & $\mathrm{S}$ & 15 & 40 \\
\hline $001 \mathrm{~A} 5$ & TAHOE CITY & $\mathrm{A}$ & $\mathrm{A}$ & $\mathrm{S}$ & $\mathrm{A}$ & 15 & 40 \\
\hline 20 & $\begin{array}{c}\text { FAIRWAY } \\
\text { TRACT }\end{array}$ & $\mathrm{N}$ & A & $\mathrm{N}$ & $\mathrm{N}$ & 0 & 0 \\
\hline 21 & $\begin{array}{l}\text { FAIRWAY } \\
\text { TRACT SA }\end{array}$ & A & A & $\mathrm{N}$ & $\mathrm{N}$ & 8 & 0 \\
\hline 22 & $\begin{array}{l}\text { FAIRWAY } \\
\text { TRACT SA }\end{array}$ & $\mathrm{N}$ & A & $\mathrm{S}$ & A & 0 & 40 \\
\hline 30 & $\begin{array}{l}\text { LOWER } \\
\text { TRUCKEE }\end{array}$ & $\mathrm{N}$ & A & $\mathrm{N}$ & $\mathrm{S}$ & 0 & 0 \\
\hline 40 & $\begin{array}{l}\text { BURTON } \\
\text { CREEK }\end{array}$ & $\mathrm{N}$ & $\mathrm{S}$ & $\mathrm{N}$ & $\mathrm{S}$ & 0 & 0 \\
\hline 50 & $\begin{array}{l}\text { ROCKY } \\
\text { RIDGE }\end{array}$ & $\mathrm{N}$ & A & $\mathrm{S}$ & $\mathrm{S}$ & 0 & 20 \\
\hline 70 & $\begin{array}{c}\text { LAKE } \\
\text { FOREST } \\
\text { GLEN }\end{array}$ & A & A & $\mathrm{N}$ & $\mathrm{N}$ & 15 & 0 \\
\hline 71 & $\begin{array}{c}\text { LAKE } \\
\text { FOREST } \\
\text { GLEN }\end{array}$ & A & A & $\mathrm{N}$ & A & 15 & 0 \\
\hline 80 & $\begin{array}{c}\text { LAKE } \\
\text { FOREST }\end{array}$ & $\mathrm{N}$ & A & $\mathrm{N}$ & $\mathrm{N}$ & 0 & 0 \\
\hline 81 & $\begin{array}{c}\text { LAKE } \\
\text { FOREST }\end{array}$ & $\mathrm{N}$ & A & $\mathrm{N}$ & $\mathrm{S}$ & 0 & 0 \\
\hline 009A1 & $\begin{array}{l}\text { LAKE } \\
\text { FOREST } \\
\text { COM. }\end{array}$ & $\mathrm{S}$ & $\mathrm{S}$ & $\mathrm{S}$ & A & 15 & 40 \\
\hline 009A2 & $\begin{array}{c}\text { LAKE } \\
\text { FOREST } \\
\text { COM. }\end{array}$ & $\mathrm{S}$ & $\mathrm{S}$ & $\mathrm{N}$ & A & 15 & 0 \\
\hline 009B0 & $\begin{array}{l}\text { DOLLAR } \\
\text { HILL }\end{array}$ & $\mathrm{S}$ & $\mathrm{S}$ & $\mathrm{N}$ & A & 15 & 0 \\
\hline 100 & $\begin{array}{l}\text { DOLLAR } \\
\text { POINT }\end{array}$ & $\mathrm{N}$ & A & $\mathrm{N}$ & $\mathrm{N}$ & 0 & 0 \\
\hline 120 & $\begin{array}{c}\text { NORTH } \\
\text { TAHOE HIGH }\end{array}$ & $\mathrm{N}$ & $\mathrm{S}$ & $\mathrm{N}$ & $\mathrm{N}$ & 0 & 0 \\
\hline 130 & $\begin{array}{l}\text { WATSON } \\
\text { CREEK }\end{array}$ & $\mathrm{N}$ & $\mathrm{N}$ & $\mathrm{N}$ & $\mathrm{N}$ & 0 & 0 \\
\hline
\end{tabular}

Notes: (A) is allowed use, $(\mathrm{S})$ is a special use and must be considered under the provisions of the particular plan area statement or community plan, and $(\mathrm{N})$ is not allowed use. The density of single family dwellings is 1 unit per parcel, and the density of commercial lots is 25 percent of a parcel's area. 


\section{Appendix 4: Python Code for the Land Use Simulation Model}

The following code and its extensive comments executes as a stand-alone version and is provided here as a professional courtesy for any developer who would like to apply the code to their own problem or location, or prove to themselves that the code is adequate and fully functional. Note that this version of the code is not an exact copy of the code behind the TIIMS-hosted decision support tool.

from random import*

from copy import deepcopy

from pg import DB

import datetime

import time

\#Tahoe Decision Support Software

\#Model coded by Ben Oldham

\#Model logic prototype developed by Ben Oldham and Will Forney

\#Model transferred to new Desktop by Will Forney, Peter Ng and Mike Gould. Will Updated Default Model Values, 1/25/2010.

\#Notes:

\#

\# The model consists of a single simulation within which there are a number of runs.

\# During the course of a simulation run, vacant parcels can stay vacant or they can transition into a variety of other landuses.

\# These landuses are LU_0001, LU_1005, LU_1006, LU_1007, LU_1011, LU_2002, LU_3000, LU_6401.

\# These transitions can occur as a result as a variety of different mechanisms.

\#Generalized Model flow:

\# Pass in the Model ID.

\# Initialize the model object:

\# Obtain the model parameter values.

\# Get PAS db data (model.PASList) that specifies what landuses and densities are allowed for the parcels in each PAS (PAS.allowedLanduseDensities).

\# Get vacant parcel db data (model.parcelList) with attributes that help determine the various possible fates of a parcel. 
\# For each parcel, identify the possible transitions and landuses that the parcel qualifies for (parcel.possibleTransitionsMasterList, parcel.landuseCounts).

\# Generate a master set of lists (model.transitionCandidatesMasterLists), one for each transition type, with each list containing the apns of the parcels that qualify for that transition type.

\# Initialize a number of analysis statistics.

\# Identify each parcels category (parcels with the same category all share functionally similar attributes and can be expected to have similar outcome probabilities).

\# Initialize the run object.

\# Perform $\mathrm{n}$ runs (where $\mathrm{n}=$ the ITERATIONS parameter)

\# At the start of each run, initialize the run (run.initRun):

\# Initialize the year and pool values, etc.

\# Refresh the working copy of the transition candidate parcel lists (thisRunTransitionCandidates) and randomize them.

\# For each parcel, copy the thisRunPossibleTransitions list from the possibleTransitionsMasterList.

\# For each year of the run:

\# Initialize the this YearTrans list (initRunYear):

\# Determine how many transitions of each type will be scheduled for the current year based on the input parameters (run.thisYearTrans) (stochastic values). For all SFH and Retirement transitions, add that number of items to the list.

MFH, Tourist and Commercial each have only a single item added to the list as they are handled with a different mechanism (pools).

\# Randomize the list.

\# Do some minor annual tasks (filterLargeCommLots, checkVacantLotEqPL).

\# Attempt to do the current year's scheduled transitions:

\# For each transition, determine the transition list, landuse, and transition type (there are special pool mechanisms for MFH, Tour and Comm).

\# If a candidate parcel exists (the length of the transition list is greater than 0 ), do the transition (if no candidates exist, then the scheduled transition is disregarded):

\# With the first parcel in the transition list:

\# For each of the transition types in the parcel thisRunPossibleTransitions, remove that parcel from the associated transition list (in thisRunTransitionCandidates).

\# Update various analysis statistics.

\# Increment the run year.

\# Now that the simulation is done, do some minor massaging of the analysis statistics to make them ready for output (calcOutputResults).

\# Print the seven analysis reports.

class TDSS(object):

\#class instantiates the Model and Run objects, runs the appropriate number of iterations, and prints the results

def init (self, modelID):

\#Starting the simulation.

\#The model object stores data and results for the entire course of the simulation (all runs).

\#The run object stores data and results for a single run of the simulation.

print "START: ", datetime.datetime.fromtimestamp(time.time()) 
model $=$ Model $($ modelID $)$

\#Initialize the Model object - get db data (paramater, pas, parcel), do initialization for the simulation.

run $=$ Run (model)

\#Initialize the Run object.

\#Now that we're initialized, we can do the simulation.

for iteration in range(model.params["ITERATIONS"]):

print "ITER = ", iteration

run.initRun()

while (run.thisYear $<=$ model.params["TOTAL_YEARS"]) :

run.initRunYear()

run.filterLargeCommLots()

run.checkVacantLotEqPL(

run.annualTransitions()

run.thisYear $+=1$

\#Simulation is finished, now print the results

model.calcOutputResults()

model.printLanduse(str(modelID))

model.printCondensedLanduse( $\operatorname{str}($ modelID))

model.printCondensedTransitions(str(modelID))

model.printLanduseByYear(str(modelID))

model.printTransitionsByYear(str(modelID))

model.printTransCandidatesByYear(str(modelID))

\#Initialize start-of-run values and transition lists.

model.printCategoryTotals(str(modelID))

\#Determine the scheduled transitions for the year.

\#Unclog the commercial "development pipeline".

\#See if the PL vacant lot equation is satisfied.

\#Attempt to process the scheduled transitions for the year.

\#Massage the analysis statistics to make them ready for output

\#Analysis report \#1.

\#Analysis report \#2.

\#Analysis report \#3.

\#Analysis report \#4.

\#Analysis report \#5.

\#Analysis report \#6.

\#Analysis report \#7.

print "END:", datetime.datetime.fromtimestamp(time.time())

class Model(object):

def init (self, modelID):

\#The model object stores data and results for the entire course of the simulation (all runs).

self.landuseNames = ("LU_0001", "LU_1005", "LU_1006", "LU_1007", "LU_1011", "LU_2002", "LU_3000", "LU_6401", "MFHUnits", "TourUnits", "CommSF") \#really a grabbag of stuff - used for the TahoeLanduseByYear report

self.transTypes = ("ALLOC_DG", "ALLOC EL", "ALLOC PL", "ALLOC PL SENSITIVE", "ALLOC WA", "ALLOC SLT", "ALLOC MFH",

"ALLOC_TOUR", "ALLOC_COMM", "RETIRE_CTC_PRIÖRITY", "RETIRE_CTC_SECONDARY", "RETIRE_NEV_PRIORITY",

"RETIRE NEV SECONDARY", "RETIRE_TRPA_PRIORITY", "RETIRE_TRPA_SECONDARY", "RETIRE_USFS PRIORITY",

"RETIRE_USFS_SECONDARY")

self.dbConn $=$ self.getDBConn()

self.params $=$ self.getFixedParams ()

\#Simulation parameters

self.params["MODEL_ID"] = modelID 
self.params.update(self.getUserParams())

self.PASList $=$ self.getPASs ()

self.parcelList = self.getParcels(self.params["IPES_THRESHOLD"])

self.dbConn.close()

self.getParcelPossibleFates ()

possibleTransitionsMasterList.

\# Also initializes the parcel landuse analysis statistic (landuseCounts) (reports \#1 \& 2).

self.transitionCandidatesMasterLists = self.popTransitionCandidates() \#A master set of lists that never change, one for each transition type.

\# Each sub-list contains a list of parcels (apn numbers) that are valid candidates for that transition type.

\# If an apn is in a particular model.transitionCandidatesMasterLists list, than the possibleTransitionsMasterList for that parcel will always include that transition

type.

\# There is always a one-to-one correspondence between the apns in a particular model.transitionCandidatesMasterLists list and the transitions in the member parcel.possibleTransitionsMasterList.

self.initParcelTransitionCounts()

self.landuseByYear = self.initLanduseByYear(

self.transitionsByYear = self.initTransitionsByYear()

self.transCandidatesByYear $=$ self.initTransCandidatesByYear()

\#6).

self.categoryTotals $=$ self.initCategoryTotals ()

self.calcParcelCatLanduseBinaryTotal()

\#Initialize the parcel transition type analysis statistic (report \#3).

\#Initialize the simulation landuse by year analysis statistic (report \#4).

\#Initialize the simulation transitions by year analysis statistic (report \#5).

\#Initialize the simulation transitions candidates by year analysis statistic (report

\#Initialize the simulation category totals analysis statistic (report \#7).

\#Calculate parcel landuseBinaryTotal values (used to help determine the parcel's

self.calcParcelCategory ()

\#For each parcel, identify the category for the parcel.

def getDBConn(self):

\#Opens a connection to the postgresql database.

\#Java version may want to read connection parameters from some sort of ini file

dbname $=$ "test $2 "$

host $=$ "localhost"

port $=5432$

options $=$ None

tty $=$ None

user $=$ "postgres $2 "$

password="QAZSE45tgb1!2@"

return $\mathrm{DB}$ (dbname, host, port, options, tty, user, password) \# returns a connection to the database

def getFixedParams(self):

\#Getting the model parameters that aren't exposed to the users for input.

\#Java version will probably read paramater values from a model_execution_parameters table using the Model ID as an identifier. 
\#Both fixed and user parameter values should probably be stored in the model_execution_parameters table.

\#Master default values should probably be stored in a static table (perhaps fixed_params).

params $=\{\}$

dbParams = self.dbConn.query ("SELECT * FROM fixed_params").dictresult()

\#params["ITERATIONS"] = dbParams[0]["iterations"]

\#The somewhat arbitrary value of 500 used in the previous version of the model is again used as a default.

params["ITERATIONS"] = 2

params["IPES_THRESHOLD"] = dbParams[0]["ipes_threshold"]

\#At some point in the run, threshold may change to zero once the PL vacant lot equation has been satisfied.

params["COMM_AREA_RATIO"] = dbParams[0]["comm_area_ratio"]

\#Ratio used to tell the net commercial area sf based on the parcel size (default set to historical value of .25)

params["MFD_UNITS_PER_ACRE"] = dbParams[0]["mfd_units_per_acre"] \#Empirically derived (default set to 18).

params["TOUR_UNITS_PER_ACRE"] = dbParams[0]["tour_units_per_acre"] \#Empirically derived (default set to 30).

return params

def getUserParams(self):

\#Getting the model parameters with user-input values.

\#Java version will probably read paramater values from a model execution parameters table using the Model ID as an identifier.

\#Both fixed and user parameter values should probably be stored in the model_execution_parameters table.

\#Master default values should probably be stored in a static table (perhaps default_user_params) and then displayed to the user.

\#Most of the values (annual housing allocation, retirement, allocation rollover pool) were determined by Will Forney based on historical averages and review of

TRPA Code of Ordinances such as the Residential Allocation Performance Table.

\#Other values were jointly arrived at by discussion Will Forney/Ben Oldham.

params $=\{\}$

\#housing allocations - all are annual numbers

params["MIN_ALLOC_DG"] $=9 \quad$ \#was 10

params["MAX ALLOC DG"] $=21 \quad$ \#was 14, then 17 for Ben's Evaluation

params["MIN_ALALOC_EL"] = $27 \quad$ \#was 73

params["MAX ALLOC EL"] = $111 \quad$ \#was 89, then 83 for Ben's Evaluation

params["MIN_ALLOC_PL"] = 18 \#was 38

params["MAX_ALLOC_PL"] $=66 \quad$ \#was 46, then 50 for Ben's Evaluation

params["MIN ALLOC WA"] = $13 \quad$ \#was 32

params["MAX_ALLOC_WA"] $=49 \quad$ \#was 40, then 38 for Ben's Evaluation

params["MIN ALLOC SLT"] = $11 \quad$ \#was 29

params["MAX_ALLOC_SLT"] $=47 \quad$ \#still 35

params["MIN ALLOC MFH"] $=30 \quad$ \#was 18

params["MAX_ALLOC_MFH"] $=50 \quad$ \#was 18

params["MIN_ALLOC_TOUR"] $=30 \quad$ \#was 5 
params["MAX_ALLOC_TOUR"] = 50

\#retirements - all are annual numbers

params["MIN RETIRE CTC"] = 86

params["MAX_RETIRE_CTC"] $=250$

params["MIN RETIRE NEV"] $=3$

params["MAX_RETIRE_NEV"] $=59$

params["MIN_RETIRE_TRPA"] $=29$

params["MAX RETIRE TRPA"] $=46$

params["MIN_RETIRE_USFS"] = 91

\#retirement priorities

params["PRIORITY_RETIRE CTC"] $=.5$

params["PRIORITY_RETIRE_NEV"] $=.5$

params["PRIORITY_RETIRE_TRPA"] $=.5$

params["PRIORITY_RETIRE_USFS"] $=.5$

\#other params

params["COMM AREA TOTAL ALLOC"] $=160000$

params["ALLOC_ROLLOVER_POOL"] $=48$

params["MIN ACRES"] = .1

params["ALLOW_SPECIAL_USE"] = False

params["START_YEAR"] $=\overline{2} 009$

params["END YEAR"] $=2028$

params["TOTĀL_YEARS"] = params["END_YEAR"] - params["START_YEAR"] + 1

return params

\section{def getPASs(self)}

\#Getting the PAS (TRPA Plan Area Statement) data from the db (the db contains data for all PASs).

PASList $=\{\}$

PASs = self.dbConn.query("SELECT * FROM pas_areas").dictresult()

for pas in PASs:

PASList[pas["pas_id"]] = PAS(self.params["ALLOW_SPECIAL_USE"], **pas)

return PASList

def getParcels(self, ipesThreshold):

\#Getting the parcel data from the $\mathrm{db}$ (the $\mathrm{db}$ contains only vacant parcels - landuse code 1 )

parcelList $=\{\}$

parcels $=$ self.dbConn.query("SELECT * FROM parcels").dictresult()

for parcel in parcels: (the model - was 40000

\#Applies to SFH, MFH and tourist parcels

\#Will parcels in PAS's with LU "S" be included? (Default inclusion is LU "A") 
parcelList[parcel["apn"]] = Parcel(self.PASList, ipesThreshold, **parcel) return parcelList

def getParcelPossibleFates(self):

\#Here, the parcels possible transitions and possible landuses are identified and stored.

\#Go through all the parcels, and, for each landuse allowed by the parcel's PAS, call the appropriate function.

\#The selected parcelPossibleLanduseXXXX function will examine the parcel to see if it meets the criteria for that landuse.

\#If it meets the criteria, the called function adds the appropriate possible transition type to the parcels possibleTransitionsMasterList.

\#Also, initialize the parcel landuse analysis statistic (reports \#1 \& 2).

\#Gives each parcel a dictionary of valid possible landuses (landuseCounts) with each value set to 0 (dictionary does not typically include the entire set of

landuses).

\#Ultimately, each value will represent the number of times that the parcel transitioned to that landuse.

landuseFunctions $=\{$ "LU_1005": self.parcelPossibleLanduse1005, "LU_1011": self.parcelPossibleLanduse1011, "LU_2002": self.parcelPossibleLanduse2002,

"LU_3000": self.parcelPossibleLanduse3000, "LU_6401": self.parcelPossibleLanduse6401\}

for apn in self.parcelList:

parcel $=$ self.parcelList[apn]

PAS = self.PASList[parcel.pas_id]

for landuse in PAS.allowedLanduseDensities.keys():

landuseFunctions.get(landuse)(parcel, PAS.allowedLanduseDensities[landuse])

parcel.landuseCounts["LU_0001"] = 0

def parcelPossibleLanduse1005(self, parcel, PASdensity):

\#MFH

\#Parcel derivedIPES must exceed 0, it must exceed the minimum size, and the calculated units must exceed 1.

\#The landuse is either 1005 (2-4 units) or 1006 (5-10 units) or 1007 (10+ units) depending on the number of units that the parcel supports.

\#Calculate unitsMFH, a new Parcel attribute which is the developable MFH units based on the min of parcel size * MFH_UNITS_PER_ACRE, or the legal max density from PASdensity

if (parcel.derivedIPES $>0$ ) and (parcel.acres >= self.params["MIN_ACRES"]):

units $=\min ($ int $($ parcel.acres * self.params["MFD_UNITS PER ACRE"]), PASdensity)

if units $>1$ :

parcel.unitsMFH = units

parcel.possibleTransitionsMasterList.append("ALLOC_MFH")

landuse $=$ parcel.mfhLanduse(units)

parcel.landuseCounts[landuse $]=0$

def parcelPossibleLanduse1011(self, parcel, PASdensity):

\#SFH

\#Parcel derivedIPES must exceed 0, and it must exceed the minimum size. 
\#The transition type depends on the jurisdiction (5 jurisdictions, including SLT).

\#Additionally, the transition type for jurisdiction PL has two types, depending on the derivedIPES (because of the PL vacant lot equation).

if (parcel.derivedIPES $>0$ ) and (parcel.acres >= self.params["MIN_ACRES"]):

if parcel.jurisdiction $==$ "PL":

if parcel.derivedIPES > self.params["IPES_THRESHOLD"]:

parcel.possibleTransitionsMasterList.append("ALLOC PL")

else:

parcel.possibleTransitionsMasterList.append("ALLOC_PL_SENSITIVE")

elif parcel.jurisdiction == "EL":

if parcel.sit:

parcel.possibleTransitionsMasterList.append("ALLOC SLT")

else:

parcel.possibleTransitionsMasterList.append("ALLOC_EL")

elif parcel.jurisdiction in ("DG", "WA"):

parcel.possibleTransitionsMasterList.append("ALLOC_" + parcel.jurisdiction)

parcel.landuseCounts["LU_1011"] = 0

def parcelPossibleLanduse2002(self, parcel, PASdensity):

\#Tourist

\#Parcel derivedIPES must exceed 0 , it must exceed the minimum size, and the calculated units must exceed 1.

\#Calculate unitsTour, a new Parcel attribute which is the developable tour units based on the min of parcel size * TOUR UNITS PER ACRE, or the legal max

density from PASdensity

if (parcel.derivedIPES $>0$ ) and (parcel.acres >= self.params["MIN_ACRES"]):

units $=\min ($ int $($ parcel.acres * self.params["TOUR_UNITS_PER_ACRE"]), PASdensity)

if units $>1$ :

parcel.unitsTour $=$ units

parcel.possibleTransitionsMasterList.append("ALLOC TOUR")

parcel.landuseCounts["LU_2002"] = 0

def parcelPossibleLanduse3000(self, parcel, PASdensity):

\#Commercial

\#Parcel derivedIPES must exceed 0

\#Calculate commFt, a new Parcel attribute which is the developable comm sf based on the parcel size * COMM_AREA_RATIO (no maximum)

SF_PER_ACRE $=43560$

if (parcel.derivedIPES $>0$ ):

feet $=$ int(parcel.acres * SF_PER_ACRE * self.params["COMM_AREA_RATIO"])

if feet $>0$ : 


\section{parcel.commFt $=$ feet}

parcel.possibleTransitionsMasterList.append("ALLOC_COMM")

parcel.landuseCounts["LU_3000"] = 0

def parcelPossibleLanduse6401(self, parcel, PASdensity):

\#Retirement

\#All parcels are eligible for retirement.

\#There are 4 retirement agencies and every parcel will be eligible for retirement by exactly 3 of these agencies.

\#All parcels throughout the Tahoe basin qualify for USFS retirement.

\#All parcels throughout the Tahoe basin qualify for TRPA retirement.

\#Nevada parcels (DG and WA) qualify for NEV retirement.

\#California parcels (EL/SLT and PL) qualify for CTC retirement.

\#Additionally, each agency is deemed to have a priority retirement policy and a secondary retirement policy.

\#All agencies are deemed to prioritize sensitive parcels (derivedIPES <= IPES_THRESHOLD).

\#Therefore, each parcel qualifies for 3 of the 8 different retirement transition types.

parcel.landuseCounts["LU_6401"] = 0

if parcel.derivedIPES $<=$ self.params["IPES_THRESHOLD"]:

parcel.possibleTransitionsMasterList.append("RETIRE USFS PRIORITY")

parcel.possibleTransitionsMasterList.append("RETIRE_TRPA_PRIORITY")

if parcel.jurisdiction in ("EL", "PL"):

parcel.possibleTransitionsMasterList.append("RETIRE CTC PRIORITY")

if parcel.jurisdiction in ("DG", "WA"):

parcel.possibleTransitionsMasterList.append("RETIRE NEV PRIORITY")

else:

parcel.possibleTransitionsMasterList.append("RETIRE USFS SECONDARY")

parcel.possibleTransitionsMasterList.append("RETIRE_TRPA_SECONDARY")

if parcel.jurisdiction in ("EL", "PL"):

parcel.possibleTransitionsMasterList.append("RETIRE_CTC_SECONDARY")

if parcel.jurisdiction in ("DG", "WA"):

parcel.possibleTransitionsMasterList.append("RETIRE_NEV_SECONDARY")

def popTransitionCandidates(self):

\#Generate a master set of lists, one for each transition type, with each list containing the apns of the parcels that qualify for that transition type.

transitionCandidates $=\{\}$

for transType in self.transTypes:

transitionCandidates[transType $]=[]$

for apn in self.parcelList:

for transType in self.parcelList[apn].possibleTransitionsMasterList: 
transitionCandidates[transType].append(apn)

return transitionCandidates

def initParcelTransitionCounts(self):

\#Initialize the parcel transition type analysis statistic (report \#3).

\#Gives each parcel a dictionary of transition types with each value set to 0 .

\#Ultimately, each value will represent the number of times that the parcel experienced that transition type.

for apn in self.parcelList:

parcel $=$ self.parcelList[apn]

for transType in self.transTypes:

parcel.transitionCounts[transType $]=0$

def initLanduseByYear(self):

\#Initialize the simulation landuse by year analysis statistic (report \#4).

\#Creates a set of lists, one for each landuse.

\#Gives landuseByYear[landuse] a list of length TOTAL YEARS with each value set to 0 .

\#Ultimately, each list will contain a set of values that represent the average number of parcels that transitioned to that landuse in that year.

\#landuseByYear[landuse][0] is the count for that landuse in year 1, while the last item in the list, landuseByYear[landuse][19] is the count for that landuse in year 20 .

landuseByYear $=\{\}$

for landuse in self.landuseNames:

landuseByYear[landuse] $=[0]$ * self.params["TOTAL YEARS"]

return landuseByYear

def initTransitionsByYear(self):

\#Initialize the simulation transitions by year analysis statistic (report \#5).

\#Creates a set of lists, one for each transition type.

\#Gives transitionsByYear[transType] a list of length TOTAL_YEARS with each value set to 0.

\#Ultimately, each list will contain a set of values that represent the average number of parcels that experienced that transition type in that year.

\#transitionsByYear[transType][0] is the count for that transition in year 1, while the last item in the list, transitionsByYear[transType][19] is the count for that transition in year 20

transitionsByYear $=\{\}$

for transType in self.transTypes:

transitionsByYear[transType] = [0]* self.params["TOTAL_YEARS"]

return transitionsByYear

def initTransCandidatesByYear(self): 
\#Initialize the simulation transitions candidates by year analysis statistic (report \#6).

\#Creates a set of lists, one for each transition type.

\#Gives transCandidatesByYear[transType] a list of length TOTAL YEARS + 1 with all values set to 0

\#except for the year zero values which are initialized with the appropriate number of candidate parcels.

\#These lists are different from the other "ByYear" lists in that there is a year zero.

\#Ultimately, each list will contain a set of values that represent the average number of candidate parcels for that transition type at the end of that year.

\#transCandidatesByYear[transType][0] is the count of available candidate parcels for that transition type at the end of year 0 ,

\#transCandidatesByYear[transType][1] is the count of available candidate parcels for that transition type at the end of year 1,

\#while the last item in the list, transCandidatesByYear[transType][20] is the count of available candidate parcels for that transition type at the end of year 20.

transCandidatesByYear $=\{\}$

for transType in self.transTypes:

transCandidatesByYear[transType] $=[0] *($ self.params["TOTAL_YEARS"] + 1)

transCandidatesByYear[transType][0] = len(self.transitionCandidatesMasterLists[transType])

return transCandidatesByYear

def initCategoryTotals(self)

\#Initialize the simulation category totals analysis statistic (report \#7).

\#Creates a set of lists, one for each category (there are 35 categories).

\#Each list contains seven values, each initially set to 0 .

\#The first list value is the count of how many parcels are associated with that category.

\#The next six values in the list are associated with the six major landuses (1005/1006/1007 is consolidated into LU MFH).

\#Ultimately, each landuse value will represent the average chance that category member parcels were transitioned into that landuse (ranges from 0 to 1 ).

categoryTotals $=\{\}$

for catID in range(35):

categoryTotals[catID] $=\{$ "Count": 0, "LU_0001": 0, "LU_MFH": 0, "LU_1011": 0, "LU_2002": 0, "LU_3000": 0, "LU_6401": 0

return categoryTotals

def calcParcelCatLanduseBinaryTotal(self):

\#For each parcel, calculate a value (landuseBinaryTotal) that will help determine the parcel's category.

\#This value stores all the valid possible landuses in a single "binary number" which is then used by calcParcelCategory.

for apn in self.parcelList:

parcel $=$ self.parcelList[apn]

for landuse in parcel.landuseCounts:

if landuse == "LU_1011":

\#SFH

parcel.landuseBinaryTotal $+=1$

if landuse in ("LU_1005", "LU_1006", "LU_1007"):

\#MFH

parcel.landuseBinaryTotal $+=\overline{2}$ 
if landuse == "LU_2002":

\#Tourist

parcel.landuseBinaryTotal $+=4$

if landuse == "LU 3000":

\#Commercial

parcel.landuseBinaryTotal $+=8$

def calcParcelCategory(self):

\#A parcel category identifies a parcel in terms of its expected behavior as determined by its attributes.

\#Parcels with the same category all share functionally similar attributes and can be expected to have similar outcome probabilities (which will generally display a bell curve distribution).

\#Categories depend on a small set of variables (jurisdiction, landuses, IPES and size).

\#Theoretically, there are some 320 categories ( 5 jurisdictions * 16 use combos $* 2$ IPES buckets $* 2$ size buckets (usually)). (Use combos $=0$ uses $(1$ way) +1 use ( 4 ways) +2 uses ( 6 ways $)+3$ uses ( 4 ways $)+4$ uses ( 1 way) $)$

\#In practice, empirical examination of the model results indicated that there some 35 identifiable categories.

\#Many of the categories have a relatively small number of members, with only ten of the categories having as many as 100 member parcels out of the 6,281 parcels modeled (smallest has 3 members).

\#The most populated categories are:

\# 1 - 1,435 parcels - various

$\# 4$ - 1,229 parcels - jur $=\mathrm{EL}($ not SLT), landuse $=\mathrm{SFH}$, IPES $>725$, size $>=.1$

$\# 6$ - 958 parcels - jur $=$ PL, landuse $=$ SFH, IPES $>725$, size $>=.1$

$\# 2$ - 856 parcels - jur $=\mathrm{SLT}(\mathrm{EL})$, landuse $=\mathrm{SFH}$, IPES $>725$, size $>=.1$

$\# 5$ - 319 parcels - jur $=$ PL, landuse $=$ SFH, IPES 1 to 725 , size $>=.1$

\# 12 - 261 parcels - jur $=$ SLT $($ EL), landuse $=\mathrm{SFH}+\mathrm{MFH}$, IPES $>725$, size $>=.1$

$\# 14$ - 252 parcels - jur $=$ EL $($ not SLT), landuse $=$ SFH + MFH, IPES $>725$, size $>=.1$

$\# 16$ - 149 parcels - jur $=\mathrm{PL}$, landuse $=\mathrm{SFH}+\mathrm{MFH}$, IPES $>725$, size $>=.1$

$\# 3$ - 117 parcels - jur $=$ SLT $(E L)$, landuse $=$ SFH + MFH, IPES 1 to 725 , size $>=.1$

$\# 8$ - 100 parcels - jur $=$ DG, landuse $=$ SFH, IPES $>725$, size $>=.1$

\#Category \#1 is a special category where, for a variety of reasons, no development ever occurs.

\#Category \#0 (23 member parcels) is also special - its parcels are so unusual that they avoid forming even a small category and so are left in the inhomogeneous category $\# 0$.

\#The below mare's nest of if statements suffice only to identify parcels in categories 1 through 34 .

\#If a parcels should happen to belong to one of the other $286(320-34=286)$ theoretical categories, the original category value of 0 is left unchanged.

\#Finally, larger acreage parcels that have an allowed commercial landuse must be treated carefully because of the tendency for large commercial parcels to get

"stuck in the pipeline".

for apn in self.parcelList:

parcel $=$ self.parcelList[apn]

if parcel.landuseBinaryTotal $==0: \quad$ \#no allowed uses

parcel.category $=1$

elif parcel.landuseBinaryTotal $==1: \quad$ \#SFH only 
if parcel.jurisdiction $==$ "EL":

if parcel.slt:

if parcel.derivedIPES > self.params["IPES_THRESHOLD"]:

parcel.category $=2$

else:

parcel.category $=3$

else:

if parcel.derivedIPES > self.params["IPES_THRESHOLD"]: parcel.category $=4$

else:

parcel.category $=5$

elif parcel.jurisdiction $==$ "PL":

if parcel.derivedIPES > self.params["IPES_THRESHOLD"]:

parcel.category $=6$

else:

parcel.category $=7$

elif parcel.jurisdiction $==$ "DG":

if parcel.derivedIPES > self.params["IPES_THRESHOLD"]:

parcel.category $=8$

else:

parcel.category $=9$

elif parcel.jurisdiction $==$ "WA":

if parcel.derivedIPES > self.params["IPES_THRESHOLD"]:

parcel.category $=10$

else:

parcel.category $=11$

elif parcel.landuseBinaryTotal $==2$ : $\quad$ \#MFH only

if parcel.jurisdiction == "EL":

if parcel.slt:

if parcel.derivedIPES > self.params["IPES_THRESHOLD"]: parcel.category $=22$

elif parcel.jurisdiction $==$ "WA":

if parcel.derivedIPES > self.params["IPES_THRESHOLD"]:

parcel.category $=23$

elif parcel.landuseBinaryTotal $==3$ :

\#SFH, MFH

if parcel.jurisdiction == "EL":

if parcel.slt:

if parcel.derivedIPES > self.params["IPES_THRESHOLD"]:

parcel.category $=12$

else: 
parcel.category $=13$

else:

if parcel.derivedIPES > self.params["IPES_THRESHOLD"]:

parcel.category $=14$

else:

parcel.category $=15$

elif parcel.jurisdiction $==$ "PL":

if parcel.derivedIPES > self.params["IPES_THRESHOLD"]:

parcel.category $=16$

elif parcel.jurisdiction $==$ "WA":

if parcel.derivedIPES > self.params["IPES_THRESHOLD"]:

parcel.category $=17$

else:

parcel.category $=18$

elif parcel.landuseBinaryTotal $==4: \quad$ \#Tour only

if parcel.jurisdiction $==$ "PL":

if parcel.derivedIPES > self.params["IPES THRESHOLD"]

parcel.category $=26$

elif parcel.landuseBinaryTotal $==8: \quad$ \#Comm only

if parcel.jurisdiction $==$ "EL":

if parcel.slt:

if parcel.derivedIPES > self.params["IPES_THRESHOLD"]:

if parcel.acres $<4$ :

parcel.category $=30$

elif parcel.acres $<12$ :

parcel.category $=0$

else:

parcel.category $=1$

else:

if parcel.derivedIPES > self.params["IPES_THRESHOLD"]:

if parcel.acres $<4$ :

parcel.category $=31$

elif parcel.acres $<12$ :

parcel.category $=0$

else:

parcel.category $=1$

elif parcel.jurisdiction $==$ "PL":

if parcel.derivedIPES > self.params["IPES_THRESHOLD"]:

parcel.category $=32$

elif parcel.jurisdiction $==$ "DG": 
if parcel.derivedIPES > self.params["IPES_THRESHOLD"]: parcel.category $=33$

else:

parcel.category $=34$

elif parcel.landuseBinaryTotal $==10: \quad \#$ MFH, Comm

if parcel.jurisdiction == "EL":

if parcel.slt:

if parcel.derivedIPES > self.params["IPES_THRESHOLD"]:

parcel.category $=24$

elif parcel.jurisdiction $==$ "WA":

if parcel.derivedIPES > self.params["IPES_THRESHOLD"]:

parcel.category $=25$

elif parcel.landuseBinaryTotal $==11: \quad$ \#SFH, MFH, Comm

if parcel.jurisdiction == "PL":

if parcel.derivedIPES > self.params["IPES_THRESHOLD"]:

parcel.category $=19$

elif parcel.landuseBinaryTotal $==12$ : $\quad$ \#Tour, Comm

if parcel.jurisdiction == "EL":

if parcel.slt:

if parcel.derivedIPES > self.params["IPES_THRESHOLD"]:

parcel.category $=27$

elif parcel.jurisdiction $==$ "PL":

if parcel.derivedIPES > self.params["IPES_THRESHOLD"]:

parcel.category $=28$

elif parcel.jurisdiction == "WA":

if parcel.derivedIPES > self.params["IPES_THRESHOLD"]:

parcel.category $=29$

elif parcel.landuseBinaryTotal $==13: \quad$ \#SFH, Tour, Comm

if parcel.jurisdiction == "PL":

if parcel.derivedIPES > self.params["IPES_THRESHOLD"]:

parcel.category $=20$

elif parcel.jurisdiction $==$ "WA":

if parcel.derivedIPES > self.params["IPES_THRESHOLD"]:

parcel.category $=21$

def calcOutputResults(self):

\#Now that the simulation is finished, we need to do some massaging of the analysis statistics to make them ready for output.

\#Loop through all the parcels to:

\# Create a LU_0001 total by subtracting the totals of other landuse totals from the number of iterations (needed because LU_0001 is not a transition - the parcel

is simply remaining in its original state). 
\# Populate the categoryTotals "Count" field with the count of member parcels.

\# With all parcel landuseCounts (used for reports \#1 \& 2):

\# For each landuse:

\# Convert landuseCounts values from raw simulation totals to percentages by dividing by the number of iterations.

\# Populate categoryTotals (report \#7) with landuses by landuse totals (LU_1005/6/7 are consolidated into LU_MFH).

\# With all parcel transitionCounts (used for report \#3):

\# Convert the transitionCounts from raw simulation totals to average values by dividing by the number of iterations.

\# With all simulation landuseByYear (report \#4):

\# For each year of each landuse:

\# Convert the landuseByYear value from a raw simulation total to an average value by dividing by the number of iterations.

\# With all simulation transitionsByYear (report \#5):

\# For each year of each transition type:

\# Convert the transitionsByYear value from a raw simulation total to an average value by dividing by the number of iterations.

\#With all simulation transCandidatesByYear (report \#6):

\# For each year of each transition type (except year 0 which is unique to this report and needs no adjustment):

\# Convert the transCandidatesByYear value from a raw simulation total to an average value by dividing by the number of iterations.

\#With all simulation categoryTotals (report \#7):

\# For each landuse of each category:

\# Convert the category landuse count from a raw simulation total to a percentage by dividing by the category count.

for apn in self.parcelList:

parcel $=$ self.parcelList[apn]

parcel.landuseCounts["LU_0001"] = self.params["ITERATIONS"] - sum(landuseCt for landuseCt in parcel.landuseCounts.values())

self.categoryTotals[parcel.category]["Count"] +=

for landuse in parcel.landuseCounts:

parcel.landuseCounts[landuse] /= float(self.params["ITERATIONS"])

if landuse in ("LU 1005", "LU 1006", "LU_1007"):

self.categoryTotals[parcel.category]["LU_MFH"] += parcel.landuseCounts[landuse]

else:

self.categoryTotals[parcel.category][landuse] $+=$ parcel.landuseCounts[landuse]

for transType in parcel.transitionCounts:

parcel.transitionCounts[transType] / = float(self.params["ITERATIONS"])

for landuse in self.landuseByYear:

for year in range(self.params["TOTAL_YEARS"]):

self.landuseByYear[landuse][year] /= float(self.params["ITERATIONS"])

for transType in self.transitionsByYear:

for year in range(self.params["TOTAL_YEARS"]):

self.transitionsByYear[transType][year] /= float(self.params["ITERATIONS"])

for transType in self.transCandidatesByYear:

for year in range $(1$, (self.params["TOTAL_YEARS"]) + 1): 
self.transCandidatesByYear[transType][year] /= float(self.params["ITERATIONS"])

for catID in self.categoryTotals:

category $=$ self.categoryTotals[catID]

for landuse in category.keys():

if landuse != "Count":

category[landuse] /= float(category["Count"])

def printLanduse(self, modelID):

\#Analysis report \#1.

\#Generates output that has one line with the probability percentage for every landuse of every parcel (where the probability is greater than 0 ).

\#If the landuse is MFH or tourist, include the appropriate number of units.

\#If the landuse is comm, include the appropriate number of developed sf.

\#This report isn't particularly useful, as it's pretty difficult to import the data into ArcMap in any useful way, however, it was the original output format agreed to with the client.

\#The total of the UsePct values should equal the number of parcels in the simulation.

self.logFile $=$ open("TahoeLanduse" + modelID + ".txt", "w")

self.logFile.write("APN,Landuse,UsePct,Units,CommSF\n")

for apn in self.parcelList:

parcel $=$ self.parcelList[apn]

for landuse in parcel.landuseCounts:

useCount $=$ parcel.landuseCounts[landuse]

if useCount $>0$

commSF $=0$

units $=0$

if landuse in ("LU_1005", "LU_1006", "LU_1007"):

units = parcel.unitsMFH

elif landuse $==$ "LU_2002":

units $=$ parcel.unitsTour

elif landuse $==$ "LU_1011":

units $=1$

elif landuse $==$ "LU_3000":

commSF $=$ parcel.commFt

parcelData $=($ apn, landuse, $\operatorname{str}($ useCount $), \operatorname{str}($ units $), \operatorname{str}($ commSF $))$

self.logFile.write(",".join(parcelData) + "\n")

self.logFile.close ()

def printCondensedLanduse(self, modelID):

\#Analysis report \#2.

\#Generates one line of data for every parcel. 
\#Data included are the probability percentages for all landuses, all units/sf, and the category code.

\#This report is similar to the LandUse report (\#1), but it is in a format that makes it much easier to import into ArcMap.

\#The sum total of all landuse totals should equal the number of parcels in the simulation.

self.logFile $=$ open("TahoeCondensedLanduse" + modelID + ".txt", "w")

self.logFile.write("APN,Category,LU_0001,LU_1005,LU_1006,LU_1007,LU_1011,LU_2002,LU_3000,LU_6401,MFHUnits,TourUnits,CommSF\n")

landuses = ("LU_0001", "LU_1005", "LU_1006", "LU_1007", "LU_1011", "LU_2002", "LU_3000", "LU_6401")

for apn in self.parcelList:

parcel $=$ self.parcelList[apn]

dataRow $=[\mathrm{apn}]$

dataRow.append(str(parcel.category))

for landuse in landuses:

if landuse in parcel.landuseCounts:

dataRow.append(str(parcel.landuseCounts[landuse]))

else:

dataRow.append("0")

if set(["LU 1005", "LU 1006", "LU 1007"]) \& set(parcel.landuseCounts):

dataRow.append( $\operatorname{str}($ parcel.unitsMF $\bar{H}))$

else:

dataRow.append("0")

if "LU_2002" in parcel.landuseCounts:

dataRow.append(str(parcel.unitsTour))

else:

dataRow.append("0")

if "LU_3000" in parcel.landuseCounts:

dataRow.append( $\operatorname{str}($ parcel.commFt))

else:

dataRow.append("0")

self.logFile.write(",".join(dataRow) + "\n")

self.logFile.close()

def printCondensedTransitions(self, modelID):

\#Analysis report \#3.

\#Generates one line of data for every parcel.

\#Data included are the probability percentages for every transition type.

\#This report is similar to the CondensedLanduse report (\#2) except that it contains transition data instead of landuse data.

\#The sum total of all transition type totals should equal the number of parcels in the simulation.

self.logFile = open("TahoeCondensedTransitions" + modelID + ".txt", "w")

fileHeader $=[" \mathrm{APN} "]$ 
fileHeader.extend(self.transTypes)

self.logFile.write(",".join(fileHeader) + "\n")

for apn in self.parcelList:

parcel $=$ self.parcelList[apn]

dataRow $=[\mathrm{apn}]$

for transType in self.transTypes:

dataRow.append(str(parcel.transitionCounts[transType]))

self.logFile.write(",".join(dataRow) + "\n")

self.logFile.close()

def printLanduseByYear(self, modelID):

\#Analysis report \#4.

\#Generates one line of data for every landuse.

\#Data included are the average number of parcels transitioning to a particular landuse, broken down by year.

\#This report contains summarized data that is not suitable for import into ArcMap.

\#The sum total of all year totals should equal the number of parcels in the simulation, as should the sum total of all the landuses.

\#The landuse totals should match the landuse totals from report \#2.

self.logFile = open("TahoeLanduseByYear" + modelID + ".txt", "w")

fileHeader $=[$ "Desc"]

for year in range(self.params["TOTAL_YEARS"]):

fileHeader.append("Y" + str(year + 1))

self.logFile.write(",".join(fileHeader) + "\n")

for landuseName in self.landuseNames:

dataRow $=[$ landuseName $]$

for year in range(self.params["TOTAL_YEARS"]):

dataRow.append(str(self.landuseByYear[landuseName][year]))

self.logFile.write(",".join(dataRow) + "'n")

self.logFile.close()

def printTransitionsByYear(self, modelID):

\#Analysis report \#5.

\#Generates one line of data for every transition type.

\#Data included are the average number of parcels experiencing a particular transition type, broken down by year.

\#This report contains summarized data that is not suitable for import into ArcMap.

\#The sum total of all year totals should equal the number of parcels in the simulation, as should the sum total of all the transition types.

\#The transition type totals should match the transition type totals from report \#3.

self.logFile = open("TahoeTransitionsByYear" + modelID + ".txt", "w")

fileHeader $=[$ "Desc" $]$ 
for year in range(self.params["TOTAL_YEARS"]):

fileHeader.append("Y" + str(year + 1))

self.logFile.write(",".join(fileHeader) + "\n")

for transType in self.transTypes:

dataRow $=[$ transType $]$

for year in range(self.params["TOTAL_YEARS"]):

dataRow.append(str(self.transitionsByY $\bar{Y}$ ear[transType][year]))

self.logFile.write(",".join(dataRow) + "\n")

self.logFile.close()

def printTransCandidatesByYear(self, modelID):

\#Analysis report \#6.

\#Generates one line of data for every transition type.

\#Data included are the number of candidate parcels for that transition type broken down by year.

\#Data values represent the number of candidates at the end of that year.

\#Accordingly, this report, unlike the by-year reports \# 4 and \#5, contains a year zero which represents the initial state of the simulation.

\#This report contains summarized data that is not suitable for import into ArcMap.

\#Neither the yearly totals or the transition type totals have any real meaning.

self.logFile = open("TahoeTransCandidatesByYear" + modelID + ".txt", "w")

\section{fileHeader $=["$ "Desc" $]$}

for year in range((self.params["TOTAL YEARS"]) + 1):

fileHeader.append("Y" + str(year))

self.logFile.write(",".join(fileHeader) + "\n")

for transType in self.transTypes:

dataRow $=[$ transType $]$

for year in range((self.params["TOTAL_YEARS"]) + 1):

dataRow.append(str(self.transCandidatesByYear[transType][year]))

self.logFile.write(",".join(dataRow) + "\n")

self.logFile.close()

def printCategoryTotals(self, modelID):

\#Analysis report \#7.

\#Generates one line of data for every category.

\#Data included are the probability percentages for all landuses as well as the count of the member parcels for that category.

\#This report contains summarized data that is not suitable for import into ArcMap (but symbolizing parcels by category within ArcMap provides excellent

feedback).

\#The sum of the counts should equal the number of parcels in the simulation.

self. $\log$ File $=$ open("TahoeCategoryTotals" + modelID + ".txt", "w") 
fileHeader $=[$ "Category" $]$

catFields = ["Count", "LU_0001", "LU_MFH", "LU_1011", "LU_2002", "LU_3000", "LU_6401"]

fileHeader.extend(catFields)

self.logFile.write(",".join(fileHeader) + "\n")

for catID in range(35):

category $=$ self.categoryTotals[catID]

dataRow $=[\operatorname{str}($ catID $)]$

for field in catFields:

dataRow.append(str(category[field]))

self.logFile.write(",".join(dataRow) + "\n")

self.logFile.close()

class PAS(object):

def_init_(self, allowSpecialUse, **kwargs):

\#The pas areas table in the $\mathrm{db}$ has been reviewed and validated so that validation does not have to occur in the model code.

\#Values in the pas_id field (primary key) are limited to valid PAS IDs.

\#Values in the landuse fields (lu_1005_allowed, lu_1011_allowed, lu_2002_allowed, lu_3000_allowed) are limited to "A", "S", and "N".

\#Whenever a lu_1005_allowed or lu_2002_allowed field has a "A" or a "S", the corresponding density field (lu_1005_density, lu_2002_density) must be an integer greater than 0 .

\#A PAS (TRPA Plan Area Statement) is a geographic unit encompassing one or more parcels.

\#Each PAS has a set of applicable permissible landuses and densities that are stored in the pas areas table.

\#These uses are defined for landuse codes 1005 (MFH), 1011 (SFH), 2002 (Tourist), 3000 (Commercial),

\#with permissions of either "A" (allowed), "S" (special use) or "N" (not allowed).

\#Landuse densities exist only for MFH and Tourist landuses (a density of 1 is assigned to other landuses

\#for purposes of the model).

\#Landuse code 1005 is really a catchall for 1005 (2-4 units), 1006 (5-10 units), 1007 (10+ units)

\#as the number of units that can built on a parcel depends on its size.

\#Landuse code 2002 includes hotels only - not the other three tourist types (2001/2003/2004).

\#Landuse 3000 includes all commercial landuses, with the "most permissive" use prevailing (if there is a

\#mix of "A", "S", and "N" in the commercial use section of the PAS document, then the PAS is set to "A").

\#Every PAS is assumed to have an allowed 6401 (retired) landuse, even though this is not explicitly in the db table.

\#Each PAS object has a allowedLanduseDensities dictionary. The keys are the landuse codes and the values are the \#allowed density for that landuse. A landuse code is only present in the dictionary if that landuse is permitted.

self.pas_id = kwargs["pas_id"]

self.allowedLanduseDensities $=\{\}$ 
if self.useAllowed(allowSpecialUse, kwargs["lu_1005_allowed"].upper()):

self.allowedLanduseDensities["LU_1005"] = kwargs["lu_1005_density"]

if self.useAllowed(allowSpecialUse, kwargs["lu_1011_allowed"].upper()):

self.allowedLanduseDensities["LU_1011"] = 1

if self.useAllowed(allowSpecialUse, kwargs["lu 2002 allowed"].upper()):

self.allowedLanduseDensities["LU_2002"] = kwargs["lu_2002_density"]

if self.useAllowed(allowSpecialUse, kwargs["lu_3000_allowed"].upper()):

self.allowedLanduseDensities["LU 3000"] = 1

self.allowedLanduseDensities["LU_6401"] = 1

def useAllowed(self, allowSpecialUse, useval):

\#Decide if a particular landuse is allowed or not based on the PAS permissions and the

\#user specified treatment of the "S" (allow special) permission.

allowed $=$ False

if (useval == "A") or ((useval == "S") and allowSpecialUse):

allowed $=$ True

return allowed

class Parcel(object):

\#The parcels table in the $\mathrm{db}$ has been reviewed and validated so that validation does not have to occur in the model code.

\#Values in the apn field (primary key) are limited to valid APNs.

\#Values in the pas_id field (foreign key) are limited to valid PAS IDs.

\#Values in the jurisdiction field are limited to DG, EL, PL, WA.

\#Values in the acres field must be greater than 0 .

\#Values in the ipes_score field must be 0 or greater.

\#Values in the land_cap field are limited to 1a, 1b, 1c, 2, 3, 4, 5, 6, 7 .

\#The slt field is a boolean field.

@staticmethod

def mfhLanduse(units):

\#Identifies the appropriate MFH landuse code based on the number of units that the parcel supports.

$$
\begin{aligned}
& \text { if units }<=4: \\
& \text { landuse = "LU_1005" } \\
& \text { elif units }<=10 \text { : } \\
& \text { landuse = "LU_1006" } \\
& \text { else: } \\
& \text { landuse = "LU_1007" } \\
& \text { return landuse }
\end{aligned}
$$


def _ init__ (self, PASList, ipesThreshold, **kwargs):

self.apn $=$ kwargs["apn"] \#Assessors Parcel Number

\#attributes that help determine the various possible fates of a parcel

self.pas id = kwargs["pas_id"] \#Foreign key to PAS data

self.jurisdiction $=$ kwargs["jurisdiction"] \#DG, EL, PL, WA (but not SLT, which is a subset of EL)

self.acres = kwargs["acres"]

self.ipes_score $=$ kwargs["ipes_score"]

\#Parcel size

\#Score indicating the "developability" of a parcel - higher scores are better suited for development.

\# Many of these values from the client are "0" - which was used to denote an IPES score of zero or to indicate that the IPES score was unknown (garbage data).

\# Hence the need to attempt to develop an IPES alternative

\# (Every one of the approximately 1800 PL parcels has an IPES score of 0 , which would otherwise make the calculation of the PL vacant lot equation problematic).

\# This score is not directly used in the model (derivedIPES is).

self.land_cap = kwargs["land_cap"]

\#IPES alternative generated from a GIS join of Bailey valued areas with parcel areas (1a, 1b, 1c, 2, 3, 4, 5, 6, 7).

\# This score is not directly used in the model (derivedIPES is).

self.slt = self.getBool(kwargs["slt"])

\#True if the parcel is a South Lake Tahoe parcel (subset of EL) (generated from a GIS join of the SLT area with parcel areas).

self.derivedIPES $=$ self.getDerivedIPES(self.ipes score, self.land cap, ipesThreshold)

\#Final IPES score that the model uses to help determine possible fates for parcel.

\#initializing some simulation logic attributes - they will be populated/calculated later on

self.possibleTransitionsMasterList $=$ [] \#The possible transitions for a parcel based on its attributes.

\# These are calculated once at the beginning of the simulation and never changes.

\# If a transition is in a parcel.possibleTransitionsMasterList, then that parcel's apn will be found in the appropriate model.transitionCandidatesMasterLists

transition list.

\# There is always a one-to-one correspondence between the transitions in parcel.possibleTransitionsMasterList and the apns in the appropriate

model.transitionCandidatesMasterLists list.

self.thisRunPossibleTransitions $=[]$

\#The current possible transitions for a parcel (in the current run).

\# Copied from the possibleTransitionsMasterList at the beginning of each run.

\# Can sometimes change in the middle of a run as events dictate.

\# Prior to a parcel being transitioned, if a transition is in a parcel.thisRunPossibleTransitions, then that parcel's apn will be found in the appropriate run.thisRunTransitionCandidates transition list.

\# Prior to a parcel being transitioned, there is always a one-to-one correspondence between the transitions in parcel.thisRunPossibleTransitions and the apns in the appropriate run.thisRunTransitionCandidates list.

\#initializing some analysis statistics attributes - they will be populated/calculated later on

self.landuseCounts $=\{\} \quad$ \#Analysis statistic that counts the number of landuses by landuse throughout the simulation (reports \#1 \& 2).

\# The keys are the landuse (LU 0001, LU 1005, LU 1006, LU 1007, LU 1011, LU 2002, LU 3000, LU 6401).

\# Landuses LU_0001, LU_1006, LU_1007 are newly used here as they did not exist in the PAS.allowedLanduseDensities.

self.transition $\bar{C}$ ounts $=\{\overline{\}}$

\#Analysis statistic that counts the number of transitions by transition type (report \#3). 
self.category $=0$

\#A parcel category identifies a parcel in terms of its expected behavior as determined by its attributes.

\# Parcels with the same category all share functionally similar attributes and can be expected to have similar outcome probabilities (which will generally display a bell curve distribution).

self.landuseBinaryTotal $=0$

\#Value that, once calculated (calcParcelCatLanduseBinaryTotal), helps determine the parcel's category

def getBool(self, boolval):

\#Needed because of a pg/python oddity.

if boolval $==$ ' $\mathrm{t}$ ':

return True

else:

return False

def getDerivedIPES(self, ipes, land_cap, ipesThreshold):

\#If the ipes_score is 0 (garbage data), use the land_cap value instead and translate the land_cap values into generalized IPES scores.

\#Otherwise, the IPES score is greater than 0 , which means that we have a good value that we can use.

\#Translation values determined by Will Forney.

\#The derivedIPES value is then used by the model to help determine possible fates for parcel.

derivedIPES $=$ ipes

if ipes $==0$ :

if land cap in ("1a", "2", "3"):

derivedIPES = ipesThreshold

elif land cap in ("4", "5", "6", "7"):

derived $\overline{I P E S}=$ ipesThreshold +1

else:\#1b, 1c

derivedIPES $=0$

return derivedIPES

\#less suitable for development (sensitive)

\#well suited for development (not sensitive)

\#development prohibited

class Run(object):

def init (self, model):

self.model $=$ model

self.thisRunTransitionCandidates $=\{\}$

self.this YearTrans $=$ []

\#\# self.model = model

\#The Run sometimes needs access to data contained within the Model object - making the Model an attribute of the Run object gives easy access to this data.

\#\# self.thisRunTransitionCandidates $=\{\}$ 
\#A mutable set of lists, one for each transition type, with each list containing the apns of the parcels that qualify for that transition type.

\# As the years of the run go by, the length of these lists is shortened as transitions occur.

\# Prior to a parcel being transitioned, if an apn is in a particular run.thisRunTransitionCandidates list, than the thisRunPossibleTransitions for that parcel will always include that transition type.

\# Prior to a parcel being transitioned, there is always a one-to-one correspondence between the apns in a particular run.thisRunTransitionCandidates list and the transitions in the member parcel.thisRunPossibleTransitions.

\#\# self.thisYearTrans = [] \#A list of transitions to be executed in the current year of the current run.

\section{def initRun(self):}

self.thisYear $=1$

\#The first year of a run is always year 1 (year 0 references refer to conditions prior to the start of the model).

self.commAllocated $=0$

\#This is a cumulative value representing the number of commercial sq. $\mathrm{ft}$. transitioned throughout the course of the run.

self.mfhRolloverPool $=0$

\#Each year, the run tries to use as many MFH allocation units as it can. The value typically hovers a little bit above zero throughout the course of the run and never drops below zero.

self.tourRolloverPool $=0$

\#Each year, the run tries to use as many Tourist allocation units as it can. The value typically hovers a little bit above zero throughout the course of the run and never drops below zero.

self.vacantLotEqSatisfiedPL $=$ False

\#See http://www.trpa.org/documents/agendas/Archive/apc_agendas/2002/apc/2002-02-13.PDF, pages 41/42.

\# Placer county is the only county that still needs to meet this requirement.

self.refreshTransitionCandidates()

\#Generate a randomized list of transitions scheduled for the year.

def refreshTransitionCandidates(self):

\#first, populate the run's thisRunTransitionCandidates lists from the model's transitionCandidates

for transType in self.model.transitionCandidatesMasterLists.keys():

self.thisRunTransitionCandidates[transType] = deepcopy(self.model.transitionCandidatesMasterLists[transType])

shuffle(self.thisRunTransitionCandidates[transType])

\#second, populate the Parcel currentPossibleTransitions from the Parcel initialPossibleTransitions

for apn in self.model.parcelList:

parcel $=$ self.model.parcelList[apn]

parcel.thisRunPossibleTransitions = deepcopy(parcel.possibleTransitionsMasterList)

\#\# def refreshTransitionCandidates(self):

\#Make a randomized set of run transition candidate parcels (thisRunTransitionCandidates) copied from the transitionCandidatesMasterLists.

\#Also, for each parcel, copy the thisRunPossibleTransitions list from the possibleTransitionsMasterList.

\#\#\#Refreshing the run thisRunTransitionCandidates list: 
\#\# for transType in self.model.transitionCandidatesMasterLists.keys():

\#\# $\quad$ self.thisRuntransitionCandidates[transType] = deepcopy(self.model.transitionCandidatesMasterLists[transType])

\#\# shuffle(self.thisRunTransitionCandidates[transType])

\#\#\#Refreshing all the parcels thisRunPossibleTransitions list:

\#\# for apn in self.model.parcelList:

\#\# parcel = self.model.parcelList[apn]

\#\# parcel.thisRunPossibleTransitions = deepcopy(parcel.initialPossibleTransitions)

\section{def OLDinitRunYear(self):}

\#No longer user - replaced by a gaussian version.

self.thisYearTrans $=[]$

\#Reset the list

self.thisYearTrans.extend(["ALLOC_DG"] * randint(self.model.params["MIN_ALLOC_DG"], self.model.params["MAX_ALLOC_DG"]))

self.thisYearTrans.extend(["ALLOC_EL"] * randint(self.model.params["MIN_ALLOC_EL"], self.model.params["MAX_ALLOC_EL"]))

self.thisYearTrans.extend(["ALLOC PL"] * randint(self.model.params["MIN ALLOC PL"], self.model.params["MAX ALLOC PL"]))

self.thisYearTrans.extend(["ALLOC_WA"] * randint(self.model.params["MIN_ALLOC_WA"], self.model.params["MAX_ALLOC_WA"]))

self.this YearTrans.extend(["ALLOC SLT"] * randint(self.model.params["MIN ALLOC SLT"], self.model.params["MAX ALLOC SLT"]))

self.thisYearTrans.extend(["RETIRE_CTC"] * randint(self.model.params["MIN_RETIRE_CTC"], self.model.params["MAX_RETIRE_CTC"]))

self.thisYearTrans.extend(["RETIRE_NEV"] * randint(self.model.params["MIN_RETIRE_NEV"], self.model.params["MAX_RETIRE_NEV"]))

self.thisYearTrans.extend(["RETIRE_TRPA"] * randint(self.model.params["MIN_RETIRE_TRPA"], self.model.params["MAX_RETIRE_TRPA"]))

self.thisYearTrans.extend(["RETIRE_USFS"] * randint(self.model.params["MIN_RETIRE_USFS"], self.model.params["MAX_RETIRE_USFS"]))

self.thisYearTrans.extend(["ALLOC MFH"])

self.thisYearTrans.extend(["ALLOC_TOUR"])

self.thisYearTrans.extend(["ALLOC $\left.\left.{ }^{-} \mathrm{COMM}^{\prime}\right]\right)$

shuffle(self.thisYearTrans)

self.mfhRolloverPool += randint(self.model.params["MIN_ALLOC_MFH"], self.model.params["MAX_ALLOC_MFH"])

self.tourRolloverPool += randint(self.model.params["MIN_ALLOC_TOUR"], self.model.params["MAX_ALLOC_TOUR"])

self.thisYearCommTarget = int(((self.model.params["COMM AREA TOTAL ALLOC"] / self.model.params["TOTAL YEARS"]) * self.thisYear) -

self.commAllocated)

def initRunYear(self):

\#Determine how many transitions of each type will be scheduled based on the input parameters (values are stochastic).

\#Stochastic values are calculated using a Gaussian (normal curve) distribution. Earlier versions of the model used a straight-line distribution, but no great difference between the methods was noted.

\#For all SFH and Retirement transitions, add that number of items to the list (thisYearTrans).

\#MFH, Tourist and Commercial each have only a single item added to the list as they are handled with a different mechanism (pools).

\#The list is then randomized and is ready for use.

\#Some notes on transitions and model logic: 
\#Transitions generally have min and max annual values based on user inputs (Comm only has a single value for the entire simulation).

\#These annual values do not change throughout the course of the simulation.

\#SFH allocations are assumed to vary due to various deduction and incentive increments.

\#Input parameter values should evenly bracket expected allocations rather than reflecting the theoretical min/max values that the TRPA code provides for \#(min annual allocation total of 78, max annual allocation total of 294, base allocation total of 150).

\#All allocations are expected to be used in the year of issue (with the exception of MFH/tourist/Comm allocations which work with an allocation pool mechanism.

self.thisYearTrans $=[]$

\#Reset the list

self.thisYearTrans.extend(["ALLOC DG"] * self.randomGauss(self.model.params["MIN ALLOC DG"], self.model.params["MAX ALLOC DG"]))

self.thisYearTrans.extend(["ALLOC_EL"] * self.randomGauss(self.model.params["MIN_ALLOC_EL"], self.model.params["MAX_ALLOC_EL"]))

self.thisYearTrans.extend(["ALLOC_PL"] * self.randomGauss(self.model.params["MIN_ALLOC_PL"], self.model.params["MAX_ALLOC_PL"]))

self.thisYearTrans.extend(["ALLOC_WA"] * self.randomGauss(self.model.params["MIN_ALLOC_WA"], self.model.params["MAX_ALLOC_WA"]))

self.thisYearTrans.extend(["ALLOC_SLT"] * self.randomGauss(self.model.params["MIN_ALLOC_SLT"], self.model.params["MAX_ALLOC_SLT"]))

self.thisYearTrans.extend(["RETIRE_CTC"] * self.randomGauss(self.model.params["MIN_RETIRE_CTC"], self.model.params["MAX_RETIRE_CTC"]))

self.thisYearTrans.extend(["RETIRE_NEV"] * self.randomGauss(self.model.params["MIN_RETIRE_NEV"], self.model.params["MAX_RETIRE_NEV"]))

self.thisYearTrans.extend(["RETIRE TRPA"] * self.randomGauss(self.model.params["MIN RETIRE TRPA"],

self.model.params["MAX_RETIRE_TRPA"]))

self.thisYearTrans.extend(["RETIRE_USFS"] * self.randomGauss(self.model.params["MIN_RETIRE_USFS"], self.model.params["MAX_RETIRE_USFS"]))

self.thisYearTrans.extend(["ALLOC_MFH"])

self.thisYearTrans.extend(["ALLOC_TOUR"])

self.thisYearTrans.extend(["ALLOC_COMM"])

shuffle(self.thisYearTrans)

self.mfhRolloverPool += self.randomGauss(self.model.params["MIN_ALLOC_MFH"], self.model.params["MAX_ALLOC_MFH"])

self.tourRolloverPool += self.randomGauss(self.model.params["MIN_ALLOC_TOUR"], self.model.params["MAX_ALLOC_TOUR"])

self.thisYearCommTarget $=\operatorname{int}((($ self.model.params["COMM_AREA_TOTAL_ALLOC"] / self.model.params["TOTAL_YEARS"]) * self.thisYear) self.commAllocated)

def randomGauss(self, minVal, maxVal):

\#Calculated a gaussian (normal curve) integer value based on a min and max, which are enforced.

\#The sigma (standard deviation) is deemed to be one third of the difference between the mean and the min.

sigma $=(\operatorname{maxVal}-\min V a l) / 6.0$

return $\mathrm{Val}=\operatorname{gauss}((\mathrm{max} V \mathrm{al}+\min \mathrm{Val}) / 2.0$, sigma $)$

if returnVal > maxVal:

return $\mathrm{Val}=\max \mathrm{Val}$

if returnVal < minVal:

returnVal $=\min V a l$

return int(round(returnVal)) 
def filterLargeCommLots(self):

\#Large lots can clog up the commercial "development pipeline", causing the total developed commercial s.f. to be much less than the allocation parameter.

\#Accordingly, if, during the current run year, a lot is too large to be developed, remove it from the ALLOC_COMM thisRunTransitionCandidates list \#(and the parcel thisRunPossibleTransitions list as well).

maxSize = self.model.params["COMM_AREA_TOTAL_ALLOC"] - self.commAllocated

for apn in self.thisRunTransitionCandidates["ALLOC_COMM"]

parcel $=$ self.model.parcelList[apn]

if parcel.commFt > maxSize:

parcel.thisRunPossibleTransitions.remove("ALLOC_COMM")

self.thisRunTransitionCandidates["ALLOC_COMM"].remove(apn)

def checkVacantLotEqPL(self):

\#See http://www.trpa.org/documents/agendas/Archive/apc_agendas/2002/apc/2002-02-13.PDF, (pdf pages 48-50).

\#Placer county is the only county that still needs to meet this requirement.

\#Once the PL vacant lot equation is satisfied, add all the ALLOC PL SENSITIVE parcels to the ALLOC PL list.

if self.vacantLotEqSatisfiedPL $==$ False:

if ((len(self.thisRunTransitionCandidates["ALLOC_PL_SENSITIVE"]) / 1667.0) < 0.2):

self.vacantLotEqSatisfiedPL $=$ True

\#now all non-zero IPES PL parcels may be developed

if (len(self.thisRunTransitionCandidates["ALLOC_PL_SENSITIVE"]) > 0):

self.thisRunTransitionCandidates["ALLOC_PL"].extend(self.thisRunTransitionCandidates["ALLOC_PL_SENSITIVE"])

shuffle(self.thisRunTransitionCandidates["ĀLLOC_PL"])

for apn in self.thisRunTransitionCandidates["ALLOC_PL_SENSITIVE"]:

parcel $=$ self.model.parcelList[apn]

parcel.thisRunPossibleTransitions.append("ALLOC PL")

parcel.thisRunPossibleTransitions.remove("ALLOC_PL_SENSITIVE")

self.thisRunTransitionCandidates["ALLOC_PL_SENSITIVE"] = []

def annualTransitions(self)

\#Go through the transitions scheduled for this year and attempt to fulfill them by calling the function appropriate to each transition type.

\#Each of these five called functions determines the appropriate transition list, landuse, and transition type and then calls the transitionParcel function which does the actual transitioning of a parcel.

\#The SFH and retirement functions will be called repeatedly whereas the MFH, Tour and Comm functions are only called once each year.

\#When all scheduled transitions have been processed, update the transCandidatesByYear analysis statistic (report \#6).

for transType in self.thisYearTrans: 


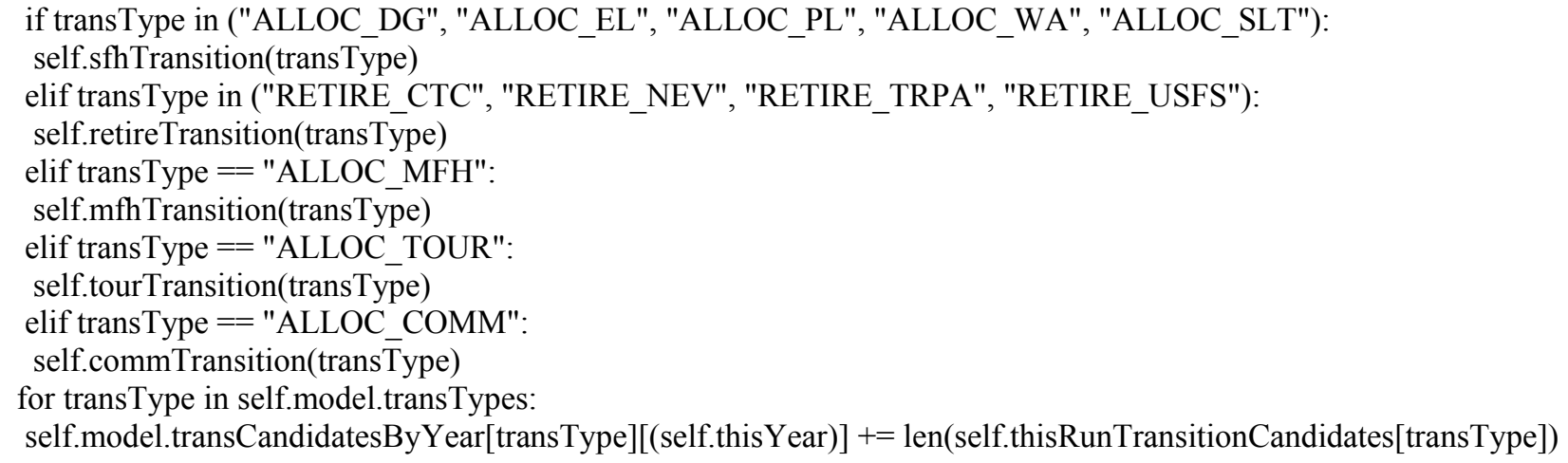

\#Retirement transitions are complicated by the fact that we have both priority and secondary retirements.

\#Accordingly, we need to determine which list and transaction type to use, priority or secondary.

\#Assuming that there are parcels in both priority and secondary lists, we randomly choose which list to use based on the user priority parameter for that retirement type.

\#If only one of the lists is populated, we will default to using that list.

priorityList $=$ self.thisRunTransitionCandidates[transType + "_PRIORITY"]

secondaryList $=$ self.thisRunTransitionCandidates[transType + "_SECONDARY"]

if (len (priorityList) $>0$ ) and (len (secondaryList) $>0)$ :

if $\operatorname{random}()<$ self.model.params["PRIORITY " + transType]:

transType $+=$ "_PRIORITY"

else:

transType $+=$ "_SECONDARY"

elif (len(priorityList) $>0)$ :

transType $+=$ "_PRIORITY"

else:

transType $+=$ " SECONDARY"

\#If the secondary list has zero length, transitionParcel will do nothing. self.transitionParcel(self.thisRunTransitionCandidates[transType], "LU_6401", transType)

def mfhTransition(self, transType): 
\#MFH transition are complicated by two things:

\#1) MFH transitions use a pool approach rather than individually scheduled transitions.

\#2) We don't know the landuse (which depends on the number of units a parcel supports).

\#Pool approach transition types attempt to transition as many parcels as possible, one after the next, without exceeding the current pool allocation.

\# Any unused pool capacity rolls forward to the next year.

\# For example, say that at the beginning of the current year the mfhRolloverPool stood at 40 and the units of the first 3 parcels in the MFH list were 16, 4, 25 .

\# The first and second parcels would be transitioned in the current year, but the third parcel would not be because the available pool units would be exceeded.

\# At the beginning of the next year, the parcel with 25 units would be first in the list and the starting pool allocation would be 20 (to which would be added the new allocation for the year).

\#To obtain the landuse, we look at units of the first parcel in the MFH list and calculate the landuse from that.

\#Because this is a pool approach transition type, there is only one MFH transition scheduled per year.

\#The landuseByYear analysis statistic for MFHUnits is also updated here.

done $=$ False

transList $=$ self.thisRunTransitionCandidates[transType]

while $(($ not done) and (len(transList $)>0))$ :

apn $=$ transList $[0]$

candidateParcel $=$ self.model.parcelList[apn]

parcelUnits $=$ candidateParcel.unitsMFH

if parcelUnits $<=$ self.mfhRolloverPool:

landuse $=$ candidateParcel.mfhLanduse(parcelUnits)

self.transitionParcel(transList, landuse, transType)

self.model.landuseByYear["MFHUnits"][(self.thisYear - 1)] += parcelUnits

self.mfhRolloverPool -= parcelUnits

else:

done $=$ True

def tourTransition(self, transType):

\#Tourist transitions use a pool approach similar to the MFH transition approach described above.

\#Tourist transitions are straightforward - the transition list, landuse, and transition type are always well defined.

\section{done $=$ False}

transList $=$ self.thisRunTransitionCandidates[transType]

while $(($ not done) and (len $($ transList $)>0))$ :

apn $=$ transList [0]

candidateParcel $=$ self.model.parcelList[apn]

parcelUnits $=$ candidateParcel.unitsTour

if parcelUnits $<=$ self.tourRolloverPool:

self.transitionParcel(transList, "LU_2002", transType)

self.model.landuseByYear["TourUnits"][(self.thisYear - 1)] += parcelUnits 
self.tourRolloverPool -= parcelUnits

else:

done $=$ True

def commTransition(self, trans Type):

\#Comm transitions use a pool approach similar to the MFH and Tour transition approachs described above.

\#One difference is that the Comm "pool" is expressed as a single value representing the allowance for the entire life of the simulation, rather than as an annual value.

\#The run attribute thisYearCommTarget is calculated as a surrogate for a pool value.

\#Another difference is that Comm is based on developed s.f. (some $25 \%$ of the parcel s.f.) rather than on parcel units.

done $=$ False

transList $=$ self.thisRunTransitionCandidates[transType]

while $(($ not done) and (len (transList) $>0))$ :

apn $=$ transList $[0]$

candidateParcel = self.model.parcelList[apn]

parcelCommSF $=$ candidateParcel.commFt

if parcelCommSF < = self.thisYearCommTarget:

self.transitionParcel(transList, "LU_3000", transType)

self.model.landuseByYear["CommSF"][(self.thisYear - 1)] += parcelCommSF

self.thisYearCommTarget $-=$ parcelCommSF

self.commAllocated $+=$ parcelCommSF

else:

done $=$ True

def transitionParcel(self, transList, landuse, transType):

\#Now that a scheduled transition has had the setup work done to identify the transition list, landuse, and transition type,

\# we can attempt to do a transition.

\#A transition can only be done if candidate parcels exist (the length of the transList is greater than 0 ).

\#If there are no candidate parcels, then there is no transition.

\#If there is a candidate parcel, then we do the transition with the first parcel in the list:

\# For each of the transition types in parcel.thisRunPossibleTransitions, remove that parcel from the associated transition list (this makes the parcel unavailable for any other transitions).

\# Update various analysis statistics.

if len(transList) $>0$ :

apn $=$ transList $[0]$

parcel $=$ self.model.parcelList[apn]

for transName in parcel.thisRunPossibleTransitions:

self.thisRunTransitionCandidates[transName].remove(apn) 
parcel.landuseCounts[landuse $]+=1$

parcel.transitionCounts[transType] $+=1$

self.model.landuseByYear[landuse][(self.thisYear - 1)] +=

self.model.transitionsByYear[transType $][($ self.thisYear - 1) $]+=1$

def main():

\#Here we are invoking the model with a hardcoded model id.

\#The final Java version should pass this value in.

$\mathrm{t}=\operatorname{TDSS}(" 1 \mathrm{a} ")$

if name $=="$ main

\#Program execution begins here

main() 